\title{
Synthese und optische Eigenschaften endständig substituierter konjugierter Polyene mit Bicyclo[2.2.2]octan als Spacer
}

Franz Effenberger* und Thomas Kesmarszky ${ }^{[1]}$

Institut für Organische Chemie der Universität Stuttgart,

Pfaffenwaldring 55, W-7000 Stuttgart 80

Eingegangen am 11. Februar 1992

Key Words: Polyenes, conjugated / Bicyclo[2.2.2]octane as spacer

Synthesis and Optical Properties of Terminally Substituted Conjugated Polyenes with Bicyclo[2.2.2]octane as Spacer

The conjugated polyenes 10, 11, and 17 respectively, with 9anthryl- and 2-tetraphenylporphyrinyl (TPP) terminal groups and a bicyclooctane unit incorporated into the polyene chain were synthesized by Wittig olefinations. In all cases it was possible to obtain the all- $(E)$ compounds from the $(E) /(Z)$-isomeric mixtures by chromatographic purification and several recrystallizations. From the UV/Vis spectra the separation of the TPP group and the polyene chain in 10, 11, and 17 can be deduced whereas in 10 and 11 the two conjugated double bonds are amalgamated into the anthryl group. In 17 the anthryl group is electronically separated from the polyene chain, and a selective excitation therefore is possible.
Untersuchungen zum Energietransfer in molekularen Systemen erlangten in den letzten Jahren zunehmende Bedeutung ${ }^{[2]}$. Neben den sehr intensiven Arbeiten zum Elektronentransfer in der Photosynthese ${ }^{[3]}$ waren die Bemühungen groß, organische Verbindungen aufzufinden, die sich für einen Energietransfer oder eine Informationsspeicherung auf molekularer Basis eignen ${ }^{[4]}$. In konjugierten Polyenen unterschiedlicher Länge, die an einem Ende einen 9-AnthrylRest und am anderen Ende einen TetraphenylporphinylRest (TPP-Rest) besitzen, konnte ein intramolekularer Energietransfer nachgewiesen werden ${ }^{[5]}$. Es war nun von Interesse $\mathrm{zu}$ untersuchen, ob und in welcher Weise der Einbau gesättigter Kohlenwasserstoffe in das konjugierte Polyen dieser Verbindungen den intramolekularen Energietransfer beeinflußt.

In der vorliegenden Arbeit berichten wir über die Synthese konjugierter Polyene mit 9-Anthryl- und 2-Tetraphenylporphyrinyl-Endgruppen, die zur Unterbrechung der Konjugation als Spacer eine Bicyclo[2.2.2] octan-Einheit enthalten. Neben der Synthese werden auch die UV/Vis-Spektren diskutiert, da sie neben der Charakterisierung der Verbindungen auch für die weitere Syntheseplanung von Modellverbindungen für den Energietransfer relevant sind. Über die Beeinflussung des Energietransfers in diesen Polyenen durch den Spacer wurde schon vorläufig berichtet ${ }^{[6]}$.

\section{Darstellung der Donor-Akzeptor-Polyene 10 und 11}

Analog zu der detailliert beschriebenen Synthese endständig 9-Anthryl- oder 2-Tetraphenylporphinyl-substituierter konjugierter Polyene ${ }^{[7]}$ wurden auch die entsprechenden mit Bicyclo[2.2.2] octan-Einheiten unterbrochenen Polyene mittels Wittig- oder Wittig-Horner-Olefinierungen hergestellt.

Bicyclo[2.2.2] octan-1,4-dicarbaldehyd (1) ${ }^{[8]}$ ließ sich zwar über eine Wittig-Olefinierung zu (Arylethenyl)bicyclooctancarbaldehyden umsetzen, aber zum einen waren die Aus-
Schema 1

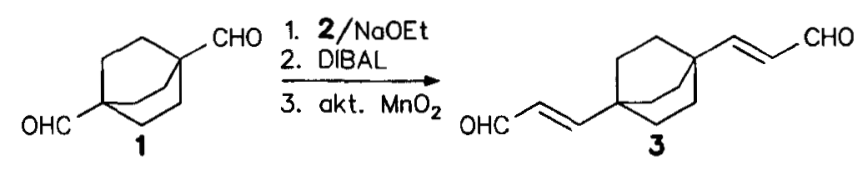

$2=\left(\mathrm{C}_{2} \mathrm{H}_{5} \mathrm{O}\right)_{2} \mathrm{POCH}_{2} \mathrm{CO}_{2} \mathrm{C}_{2} \mathrm{H}_{5}$<smiles></smiles><smiles>[R]C=CC=CC12CCC(C=CC=CC34CCC(C=CC=C[CH2+])(CC3)CC4)(CC1)CC2</smiles>

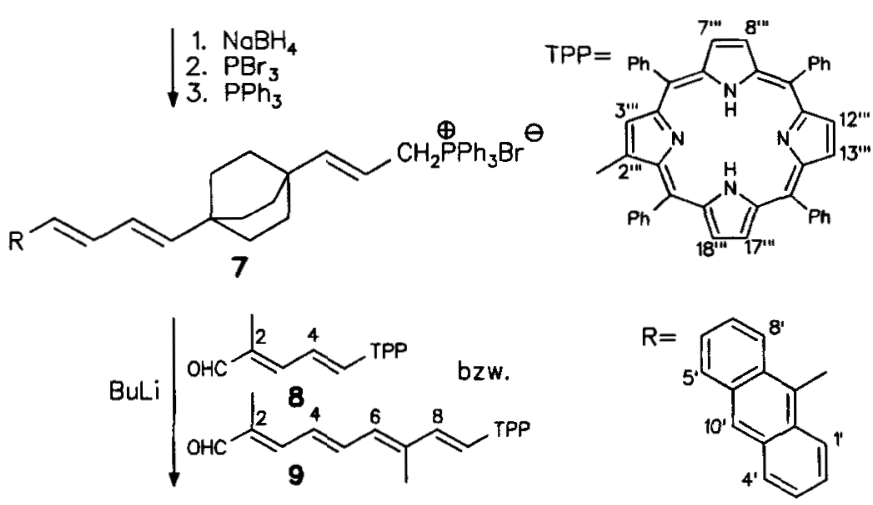<smiles>[R]C=CC=CC12CCC(C=CC=CC(C)=CC=CC=CC=CC(C)=CC=CC=C(C)C=CC=CC=CC(C)C)(CC1)CC2</smiles> 
beuten sehr gering und zum anderen konnte die AldehydGruppe am Brückenkopf nicht zum Alkohol reduziert und damit nicht weiter in das für die Wittig-Reaktion erforderliche Phosphonium-Salz übergeführt werden. Um diese wohl durch sterische Hinderung bedingten Nachteile auszuschlieBen, haben wir durch Umsetzung von 1 mit Ethoxycarbonylmethylphosphonsäure-diethylester (2), anschließende Hydrierung mit Diisobutylaluminiumhydrid (DIBAL) und selektive Oxidation des dabei erhaltenen Diols mit aktiviertem $\mathrm{MnO}_{2}$ den zu 1 vinylogen $\alpha, \beta$-ungesättigten Dialdehyd $3[(E) /(Z)>99 \%]$ dargestellt ${ }^{[9-13]}$. Die invers geführte Wittig-Reaktion von $\mathbf{3}$ mit dem aus [(9-Anthryl)methyl] triphenylphosphoniumbromid (4) ${ }^{[14]}$ mit BuLi entstehenden Ylid lieferte ein Produktgemisch aus dem Aldehyd 5 und dem symmetrischen Kopplungsprodukt 6, aus dem 5 mit einem all-(E)-Isomerenanteil $>99 \%$ als Hauptprodukt chromatographisch abgetrennt wurde. Zur Überführung von 5 in das Phosphonium-Salz 7 wurde zuerst mit Natriumborhydrid reduziert, der entstandene Alkohol dann an Kieselgel chromatographiert, nach bekannten Methoden bromiert ${ }^{[15]}$ und mit Triphenylphosphan in Toluol zu 7 umgesetzt. Nach viermaligem Umkristallisieren wird 7 mit einem all-(E)-Anteil von $>99 \%$ erhalten.

Die TPP-Verbindungen 8 und 9 wurden analog Lit. ${ }^{[7]}$ dargestellt.

Die gut zugängliche Ausgangsverbindung TPP-CHO ${ }^{[16]}$, von der Wittig-Reaktionen zu Vinyl-substituierten TPP-Derivaten bekannt sind ${ }^{[17]}$, wurde mit (4,4-Dimethoxy-3-methyl2-butenyl)triphenylphosphoniumchlorid ${ }^{[18]}$ zum 5-TPPpentadienal 8 umgesetzt. Nach Chromatographie und Umkristallisieren betrug der all- $(E)$-Anteil in $8>99 \%$. Die Darstellung des Nonatetraenals 9 mit einem all-(E)-Isomerenanteil $>99 \%$ wurde bereits beschrieben ${ }^{[7]}$.

Durch Wittig-Reaktion des Phosphonium-Salzes $7 \mathrm{mit}$ den TPP-Polyenalen 8 und 9 konnten die gewünschten Anthracen-TPP-Spacer-Polyene $\mathbf{1 0}$ und $\mathbf{1 1}$ erhalten werden. Die Reaktionen verliefen erheblich langsamer als die üblichen Wittig-Olefinierungen; so waren für die Darstellung von 1023 Stunden und für die von 1118 Stunden Reaktionszeit erforderlich. Trotzdem war in beiden Fällen die Reaktion noch unvollständig, so daß neben den Zielverbindungen 10 bzw. 11 nicht umgesetzte TPP-Polyenale vorhanden waren, die chromatographisch abgetrennt werden mußten; 10 und 11 liegen als $(E) /(Z)$-Isomerengemische vor, aus denen die dominierenden all- $(E)$-Isomere durch Mitteldruckchromatographie abgetrennt und mit einem all-(E)Anteil von $>95 \%$ bei 10 und $>97 \%$ bei 11 erhalten wurden.

\section{UV/Vis-Spektren von 10 und 11}

Die Absorptionsspektren von 10 und 11 (Abb. 1) weisen wie erwartet endgruppenähnliche Banden auf. Der langwellige Bereich von $\lambda=400-700 \mathrm{~nm}$ wird von der Absorption des Porphyrin-Restes dominiert. Eine Auftrennung in $\mathrm{S}_{2^{-}}$ (425-nm-)- und $\mathrm{S}_{1}-(520-700-\mathrm{nm})$-Übergänge ist möglich. Die Polyen-Absorption erfolgt in Abhängigkeit von der Kettenlänge bei Wellenlängen von $\lambda=320-490 \mathrm{~nm}$. Die Ver- längerung des konjugierten Systems zwischen BicyclooctanSpacer und Porphyrin-Rest um zwei Doppelbindungen bewirkt eine deutliche bathochrome Verschiebung und führt durch die vergrößerte Extinktion zum Auftreten einer Schulter bei $\lambda=370 \mathrm{~nm}$. Interessant ist der unterschiedliche Einfluß der Kettenverlängerung auf die beiden durch den Spacer getrennten Molekülteile: Während die Vergrößerung der Konjugationslänge auf der Porphyrin-Seite zu einer Abnahme im Bereich der $S_{2}$ - und einer Zunahme im Bereich der $S_{1}$-Absorption führt, bleibt die Anthracen-Bande bei $\lambda=260 \mathrm{~nm}$ nahezu unverändert. Dies verdeutlicht die gute spektroskopische Trennung beider Molekülteile durch den Spacer. Eine weitere Konsequenz dieser Trennung ist die Ausbildung einer kurzwellig absorbierenden Dien-Struktur auf der Anthracen-Seite der Moleküle. Die starke Wechselwirkung zwischen Anthracen und Dien führt zur Ausbildung eines Gesamt- $\pi$-Systems, das spektroskopisch nicht mehr in .Anthryl- bzw. Dien-Absorption aufgelöst werden kann.

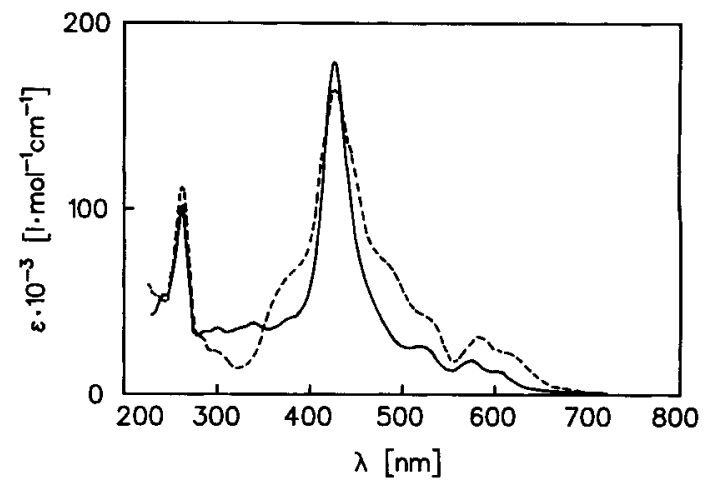

Abb. 1. UV/Vis-Absorptionsspektren der Verbindungen $10(-)$ $\left(c=0.84 \cdot 10^{-5} \mathrm{M}\right)$ und $11(---)\left(c=0.35 \cdot 10^{-5} \mathrm{M}\right)$ in $\mathrm{Di}-$ chlormethan bei $25^{\circ} \mathrm{C}$

Eine Verlängerung des Polyen-Teils zwischen Anthracen und Spacer sollte eine Verminderung dieser Wechselwirkung zur Folge haben und zu einer den nicht durch Spacer unterbrochenen Modellverbindungen ${ }^{[S]}$ vergleichbaren selektiven Anregbarkeit des Anthryl-Substituenten führen. Aus diesem Grund haben wir die Verbindung 17 dargestellt, bei der sowohl zwischen dem Anthryl-Substituenten und dem Spacer als auch zwischen dem Tetraphenylporphyrinyl-Rest und dem Spacer jeweils vier konjugierte Doppelbindungen eingeschoben sind.

\section{Darstellung des Donor-Akzeptor-Polyens 17}

In Schema 2 ist die Darstellung der Modellverbindung 17 zusammengefaßt.

Die Ausgangsverbindung 12 ist nach Lit. ${ }^{[19]}$ zugänglich und wurde mit $45 \%$ Ausbeute und einem all- $(E)$-Isomerenanteil von $>99 \%$ isoliert. Entsprechend Schema 1 wurde 12 in das Phosphonium-Salz 13 [all-(E)-Anteil >99\%] übergeführt ${ }^{[15]}$ und das aus 13 gebildete Ylid in einer inversen Wittig-Reaktion mit dem Dialdehyd 3 umgesetzt. Aus dem Produktgemisch wurde neben dem symmetrischen Dikopplungsprodukt 14 der Aldehyd 15 als Hauptprodukt nach 
chromatographischer Abtrennung mit 15\% Ausbeute erhalten; 15 lag als $(E) /(Z)$-Isomerengemisch vor, dessen HPLC-Analyse 4 Isomere im Verhältnis 44:29:18:8 zeigte, die durch MPLC getrennt wurden: Neben dem all- $(E)$-Isomeren als Hauptprodukt (10\%) haben wir das $(3 Z, 5 Z)$-Isomer mit $0.2 \%$ sowie das (3Z)-Isomer mit $4 \%$ Ausbeute nachgewiesen. Das vierte Isomer lag nur in Spuren vor.

Schema 2

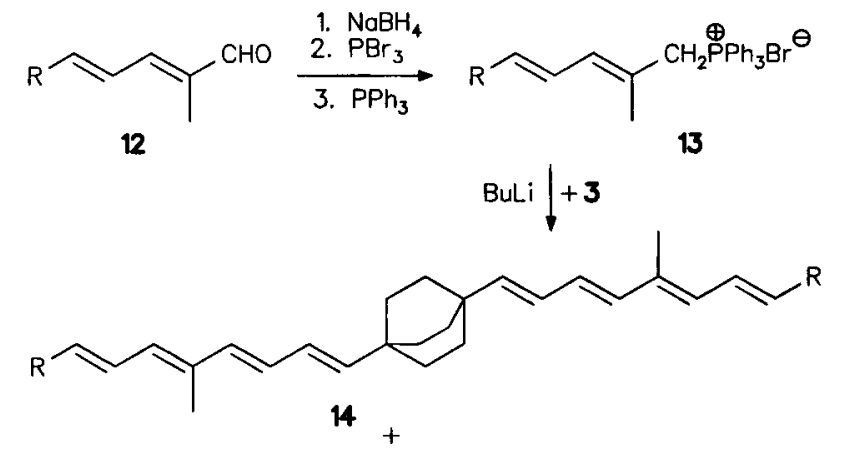<smiles>[R]C=C/C=C(C)\C=C\C=C\C12CCC(/C=C/C=O)(CC1)CC2</smiles>

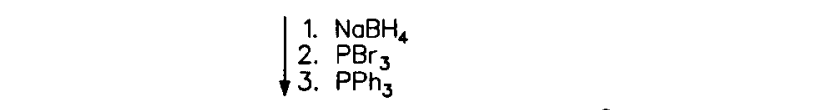<smiles>[R]C=CC=C(C)C=CC=CC12CCC(C=CC[Pb]Br)(CC1)CC2</smiles><smiles>[R]C=CC=C(C)C=CC=CC12CCC(C=CC=CC(C)=CC=C[In](C)C)(CC1)CC2(C)[13C](C)(C)C</smiles>

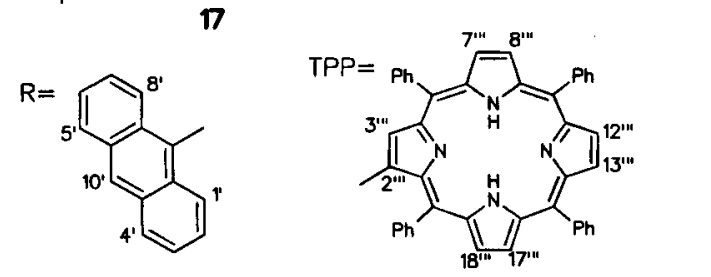

Das all-(E)-Isomere von 15 wurde in bekannter Weise zum Phosphonium-Salz 16 umgesetzt, wobei ein $(E) /(Z)$-Isomerenverhältnis für 16 von $70: 30$ erhalten wird. Im letzten Syntheseschritt wurde unter den Bedingungen der WittigReaktion das Ylid von 16 mit dem TPP-Pentadienal 8 zum Polyen-Anthracen-Bicyclooctan-Tetraphenylporphyrin 17 umgesetzt (Ausb. 10\%). Wegen der thermischen Labilität wurde 16 in Ether suspendiert und bei $0^{\circ} \mathrm{C}$ mit BuLi deprotoniert. Die Zielverbindung 17 wurde nach chromatographischer Reinigung als schwarzer Feststoff erhalten; nach Abtrennen des $(Z)$-Isomeren betrug der all- $(E)$-Isomerenanteil von $17>99 \%$.

\section{UV/Vis-Spektrum von 17}

Das Absorptionsspektrum von 17 (Abb. 2) ist im langwelligen Bereich bei $\lambda=400-700 \mathrm{~nm}$ erwartungsgemä $\beta$ nahezu identisch mit dem Absorptionsspektrum von 10. Die Anthryl-Absorption in 17 gegenüber der von 10 wird dagegen durch die Kettenverlängerung zwischen BicyclooctanSpacer und dem Anthracen stark verändert. Die Extinktion bei $\lambda=258 \mathrm{~nm}$ verringert sich und liegt mit $\varepsilon \approx 800001$. $\mathrm{cm}^{-1} \cdot \mathrm{mol}^{-1}$ in einem den nicht durch Spacer unterbrochenen Modellverbindungen ${ }^{[5]}$ vergleichbaren Bereich. Bei $\lambda=324$ und $338 \mathrm{~nm}$ treten dagegen zwei zusätzliche Banden auf, die der Wechselwirkung zwischen Anthracen und Polyen zugeschrieben werden müssen. Ähnliche Banden finden sich auch in den Spektren von 10 und 11. Die Kettenverlängerung um 2 Doppelbindungen zwischen Anthracen und dem Spacer bewirkt dabei eine bathochrome Verschiebung dieser Banden um ca. $40 \mathrm{~nm}$.

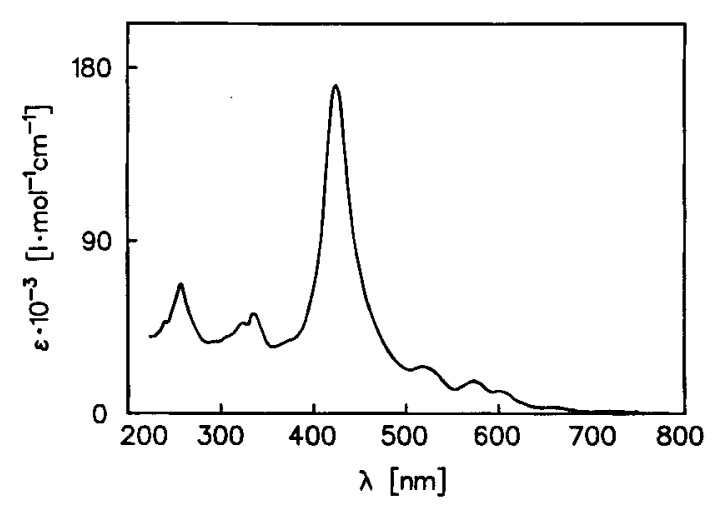

Abb. 2. UV/Vis-Absorptionsspektrum von Verbindung 17 ( $c=$ $0.62 \cdot 10^{-5} \mathrm{M}$ ) in Dichlormethan bei $25^{\circ} \mathrm{C}$

\section{Gezielte Darstellung der Verbindungen 5 und 15}

Da wir bisher die für die Synthese benötigten Aldehyde 5 und 15 jeweils nur im Produktgemisch mit 6 bzw. 14 erhalten konnten, waren wir bestrebt, diese Verbindungen gezielt darzustellen. Dies gelang durch die Blockierung einer der beiden Aldehyd-Funktionen in $\mathbf{3}$ durch Acetalisierung. Da die Substanzen zur Reinigung an saurem Kieselgel chromatographiert wurden, haben wir 2,2-Dimethyl-1,3-propandiol zur Acetalisierung eingesetzt. Das gebildete cyclische Acetal $18[(E) /(E)>99 \%]$ ist gegenüber schwachen Säuren relativ stabil und wird deshalb häufig in der Polyenchemie verwendet ${ }^{[20]}$.

Das Diacetal 18 wurde in Methanol/Wasser unter Trifluoressigsäure-Katalyse bei Raumtemp. selektiv gespalten. Aus dem erhaltenen Produktgemisch wurde nach zweimaliger Chromatographie das Monoacetal 19 mit 35\% Ausbeute und $>99 \%(E) /(E)$-Anteilen erhalten. Durch Umsetzung von 19 mit den Yliden der entsprechenden Phosphonium-Salze und anschließende Spaltung des dabei entstehenden Acetals in Dichlormethan/Ethanol/Wasser unter Trifluoressigsäure-Katalyse haben wir selektiv die Verbindungen $5[$ all $-(E)>99 \%]$ bzw. 15 [all $-(E)>97 \%]$ mit guten Ausbeuten erhalten. 
Umfassende spektroskopische Untersuchungen der in dieser Arbeit beschriebenen Donor-Akzeptor-substituierten Polyene, unter besonderer Berücksichtigung quantitativer Aspekte des Energietransfers, werden zur Zeit durchgeführt.

Schema 3

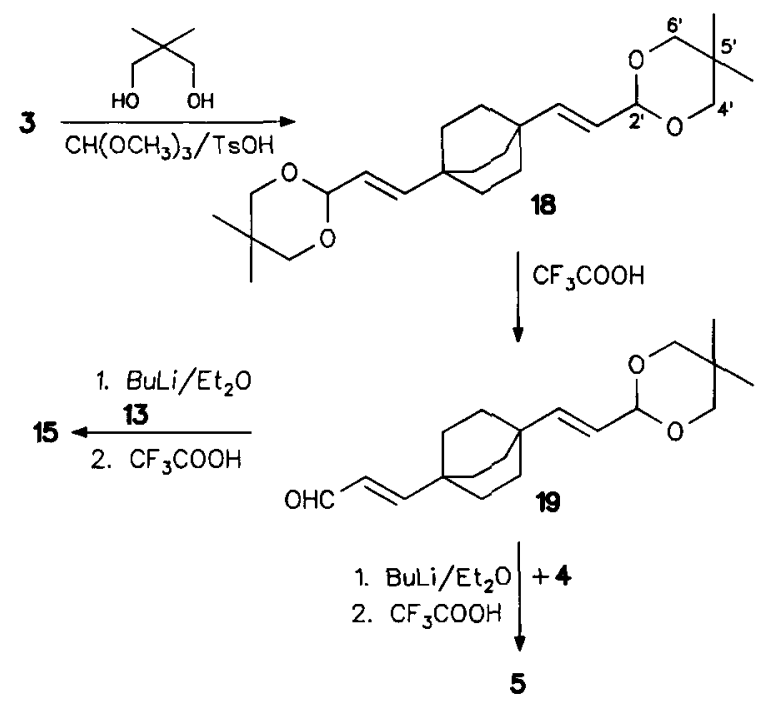

Wir danken der Deutschen Forschungsgemeinschaft (SFB 329) und dem Fonds der Chemischen Industrie für die Unterstützung dieser Arbeit sowie der BASF für die Bereitstellung wichtiger Ausgangsverbindungen.

\section{Experimenteller Teil}

${ }^{1}$ H-NMR: Varian T 60, A 60 sowie Bruker WP 80, AC $250 \mathrm{~F}$, CXP 300, AM 400; TMS als interner Standard. - UV/Vis: PerkinElmer Lambda 7. - EI-MS: Varian Mat 711. - Präparative Säulenchromatographie: Glassäulen verschiedener Größe gepackt mit Kieselgel A 60, Korngröße 0.032-0.063 mm (Riedel-de Haën). HPLC: Spektrophotometer Uvikon 720 LC (Kontron), Pumpe LDC-Milton-Roy Constametric Model III, Säule $(250 \times 4.6 \mathrm{~mm})$ mit SI $1007 \mu \mathrm{m}$ (Regu Hi-Chrom) sowie Säulen $(200 \times 4 \mathrm{~mm}) \mathrm{mit}$ Nucleosil 100-5, 5 CN, $5 \mathrm{NO}_{2}$ (Macherey-Nagel). - MPLC: Säule $(40 \times 2 \mathrm{~cm})$ mit Kieselgel LiChroprep Si 60, Korngröße 0.015-0.025 mm (Merck), 6850 Böden sowie Säule $(60 \times 4 \mathrm{~cm})$ mit Kieselgel Nucleosil $\mathrm{NO}_{2}$, Korngröße $0.015-0.025 \mathrm{~mm}(\mathrm{Ma}-$ cherey-Nagel), 2800 Böden. - Alle Lösungsmittel wurden getrocknet und destilliert eingesetzt.

Bicyclo[ 2.2.2Joctan-1,4-dicarbaldehyd (1): Nach Lit. ${ }^{\left[{ }^{[1}\right.}$.

Ethoxycarbonylmethylphosphonsäure-diethylester (2): Nach Lit. ${ }^{[10]}$.

Darstellung von 1,4-Bis[2-formylethen-1-yl]bicyclo[2.2.2]octan (3)

1) 1.4-Bis/2-(ethoxycarbonyl)ethen-1-yl]bicyclo[2.2.2]octan: In einem Dreihalskolben werden zu $0.56 \mathrm{~g}(3.40 \mathrm{mmol}) 1$ und $3.02 \mathrm{~g}$ (13.5 mmol) 2 bei $60^{\circ} \mathrm{C} 15 \mathrm{ml}(15.0 \mathrm{mmol})$ einer $1 \mathrm{~N}$ Lösung von Natriumethanolat in Ethanol mittels einer Spritze gespritzt. Nach 18stdg. Rühren bei $60^{\circ} \mathrm{C}$ wird die Reaktionslösung i. Vak. eingeengt, mit Petrolether versetzt und $2 \mathrm{~h}$ unter Rückfluß erhitzt. Die trübe Mischung wird filtriert, das Filtrat i.Vak. auf $5 \mathrm{ml}$ eingeengt und an Kieselgel mit Dichlormethan chromatographiert; Ausb. $0.61 \mathrm{~g}$ (59\%), Schmp. $58^{\circ} \mathrm{C}$ (Dichlormethan). - (E)-Isomere: ${ }^{1} \mathrm{H}-\mathrm{NMR}$
$\left(\mathrm{CDCl}_{3}\right): \delta=6.85\left(\mathrm{~d}, J_{2,1}=15.98 \mathrm{~Hz}, 2 \mathrm{H}, 2-\mathrm{H}\right), 5.66(\mathrm{~d}, 2 \mathrm{H}, 1-\mathrm{H})$, 4.16 (q, $\left.4 \mathrm{H}, \mathrm{OCH}_{2} \mathrm{CH}_{3}\right), 1.59$ (s, $\left.12 \mathrm{H}, \mathrm{CH}_{2}\right), 1.28$ (t, $6 \mathrm{H}, \mathrm{OCH}_{2} \mathrm{CH}_{3}$ ).

\section{$\mathrm{C}_{18} \mathrm{H}_{26} \mathrm{O}_{4}$ (306.4) Ber. C $70.56 \mathrm{H} 8.55$ Gef. C $70.79 \mathrm{H} 8.58$}

2) 1,4-Bis[2-(hydroxymethyl)ethen-1-yl]bicyclo[2.2.2]octan: In einem Dreihalskolben mit Tropftrichter und Sicapent-Trockenrohr werden bei $-78^{\circ} \mathrm{C}$ zu $3.60 \mathrm{~g}(11.7 \mathrm{mmol})$ 1,4-Bis[2-(ethoxycarbonyl)ethenyl] bicyclo[2.2.2] octan in $20 \mathrm{ml}$ Tetrahydrofuran (THF) langsam $50 \mathrm{ml}(50 \mathrm{mmol})$ einer $1 \mathrm{~N}$ Lösung von Diisobutylaluminiumhydrid (DIBAL) in THF getropft. Nach 20stdg. Rühren bei $-78^{\circ} \mathrm{C}$ werden langsam $20 \mathrm{ml}$ konz. $\mathrm{HCl} /$ Methanol $(1: 1)$ zugesetzt. Man läßt die Reaktionslösung auf Raumtemp. erwärmen, gießt auf Eis/Wasser, extrahiert dreimal mit Dichlormethan, trocknet die vereinigten Extrakte mit Magnesiumsulfat und engt i.Vak. ein. Der Rückstand wird aus Toluol umkristallisiert; Ausb. $0.98 \mathrm{~g}(38 \%)$, Schmp. $119-120^{\circ} \mathrm{C}$. $-(E)$-Isomere: ${ }^{1} \mathrm{H}$-NMR $\left(\mathrm{CDCl}_{3}\right): \delta=5.59$ $\left(\mathrm{d}, J_{2,1}=15.80 \mathrm{~Hz}, 2 \mathrm{H}, 2-\mathrm{H}\right), 5.48\left(\mathrm{dt}, J_{1, \mathrm{CH}_{2}}=5.55 \mathrm{~Hz}, 2 \mathrm{H}, 1-\mathrm{H}\right)$, $4.07\left(\mathrm{dd}, J_{\mathrm{CH}_{2,2}}=0.86 \mathrm{~Hz}, 4 \mathrm{H}, \mathrm{CH}_{2} \mathrm{OH}\right), 1.62(\mathrm{~s}, 2 \mathrm{H}, \mathrm{OH}), 1.51$ (s, $\left.12 \mathrm{H}, \mathrm{CH}_{2}\right)$.

$$
\mathrm{C}_{14} \mathrm{H}_{22} \mathrm{O}_{2} \text { (222.3) Ber. C } 75.63 \mathrm{H} 9.97 \text { Gef. C } 75.51 \text { H } 9.96
$$

3) In einem Zweihalskolben werden $3.00 \mathrm{~g}$ (13.5 mmol) 1,4-Bis[2-(hydroxymethyl)ethen-1-yl]bicyclo[2.2.2]octan und $10 \mathrm{~g}$ akt. $\mathrm{MnO}_{2}$ in $120 \mathrm{ml}$ Dichlormethan $8 \mathrm{~h}$ bei Raumtemp. gerührt. Nach Beendigung der Reaktion (dünnschichtchromatographisch bestimmt) wird festes $\mathrm{MnO}_{2}$ abfiltriert und mehrmals mit Dichlormethan und Diethylether gewaschen. Das Filtrat wird getrocknet, i. Vak. eingeengt und der Rückstand aus Toluol umkristallisiert; Ausb. $1.50 \mathrm{~g}(51 \%)$ 3, Schmp. $152-153^{\circ} \mathrm{C}$. - (E)-Isomere: ${ }^{1} \mathrm{H}$ NMR $\left(\mathrm{CDCl}_{3}\right): \delta=9.49\left(\mathrm{~d}, J_{\mathrm{CHO}, 1}=7.73 \mathrm{~Hz}, 2 \mathrm{H}, \mathrm{CHO}\right), 6.71(\mathrm{~d}$, $\left.J_{2,1}=15.92 \mathrm{~Hz}, 2 \mathrm{H}, 2-\mathrm{H}\right), 6.0(\mathrm{dd}, 2 \mathrm{H}, 1-\mathrm{H}), 1.67\left(\mathrm{~s}, 12 \mathrm{H}, \mathrm{CH}_{2}\right)$.

$\mathrm{C}_{14} \mathrm{H}_{18} \mathrm{O}_{2}$ (218.3) Ber. C 77.03 H 8.31 Gef. C 77.22 H 8.47

[(9-Anthryl)methyl]triphenylphosphoniumbromid (4): Nach Lit. ${ }^{[14]}$.

Umsetzung von 4 mit 3: Die Reaktion wird in einem Dreihalskolben, versehen mit Rückflußkühler mit Sicapent-Trockenrohr, Tropftrichter und Septum, durchgeführt. Der Tropftrichter ist über ein um $90^{\circ}$ abgewinkeltes Glasrohr mit einem Zweihalskolben verbunden. In diesem Kolben werden zu $3.66 \mathrm{~g}(6.90 \mathrm{mmol}) 4$ in $20 \mathrm{ml}$ Diethylether $6.80 \mathrm{ml}(6.80 \mathrm{mmol})$ einer $1 \mathrm{M}$ Lösung von BuLi in Hexan durch ein Septum gespritzt, und die Mischung wird einige Zeit gerührt. Durch Drehen des Kolbens wird die Lösung des gebildeten Ylids in den Tropftrichter übergeführt und innerhalb $2 \mathrm{~h}$ bei Raumtemp. zu $1.50 \mathrm{~g}(6.90 \mathrm{mmol}) 3$ in $100 \mathrm{ml}$ 1,2-Dichlorethan im Dreihalskolben getropft. Nach 2stdg. Rühren wird auf Wasser gegossen und die organische Phase abgetrennt. Die wäßrige Phase wird mit Dichlormethan extrahiert, die vereinigten organischen Phasen werden getrocknet und eingeengt. Der Rückstand wird an Kieselgel mit Dichlormethan chromatographiert. Die Rohprodukte werden aus Dichlormethan/Methanol oder $n$-Hexan umkristallisiert und i.Hochvak. getrocknet; Ausb. $1.08 \mathrm{~g}$ (39\%) 5, Schmp. $198^{\circ} \mathrm{C}$ und $0.06 \mathrm{~g}(1.5 \%) 6$, Schmp. $232^{\circ} \mathrm{C}$ - $-(E)$-Isomere von 5 ${ }^{1} \mathrm{H}-\mathrm{NMR}\left(\mathrm{CDCl}_{3}\right): \delta=9.50\left(\mathrm{~d}, J_{\mathrm{CHO}, 1^{\prime \prime}}=7.76 \mathrm{~Hz}, 1 \mathrm{H}, \mathrm{CHO}\right), 8.36$ (s, 1 H, 10'-H), $8.31-8.27\left(\mathrm{~m}, 2 \mathrm{H}, 1^{\prime}-, 8^{\prime}-\mathrm{H}\right), 8.01-7.96(\mathrm{~m}, 2 \mathrm{H}$, $\left.4^{\prime}-, 5^{\prime}-\mathrm{H}\right), 7.48-7.43\left(\mathrm{~m}, 4 \mathrm{H}, 2^{\prime}-, 3^{\prime}-, 6^{\prime}-, 7^{\prime}-\mathrm{H}\right), 7.29\left(\mathrm{~d}, J_{1,2}=\right.$ $15.94 \mathrm{~Hz}, 1 \mathrm{H}, 1-\mathrm{H}), 6.73\left(\mathrm{~d}, J_{2^{\prime \prime}, 1^{\prime \prime}}=15.89 \mathrm{~Hz}, 1 \mathrm{H}, 2^{\prime \prime}-\mathrm{H}\right), 6.57(\mathrm{dd}$, $\left.J_{2,3}=10.38 \mathrm{~Hz}, 1 \mathrm{H}, 2-\mathrm{H}\right), 6.02\left(\mathrm{dd}, 1 \mathrm{H}, 1^{\prime \prime}-\mathrm{H}\right), 5.77\left(\mathrm{~d}, J_{4,3}=\right.$ $15.38 \mathrm{~Hz}, 1 \mathrm{H}, 4-\mathrm{H}), 1.69$ (s, $12 \mathrm{H}, \mathrm{CH}_{2}$ ).

\section{$\mathrm{C}_{29} \mathrm{H}_{28} \mathrm{O}$ (392.5) Ber. C 88.73 H 7.19 Gef. C 88.48 H 7.04}

(E)-Isomere von 6: ' ${ }^{1} \mathrm{H}-\mathrm{NMR}\left(\mathrm{CDCl}_{3}\right)$ : $\delta=8.36\left(\mathrm{~s}, 2 \mathrm{H}, 10^{\prime}-\mathrm{H}\right)$ $8.35-8.30$ (m, 4H, 1'-, 8'-H), 8.01-7.96 (m, 4H, 4'-, 5'-H), $7.49-7.44\left(\mathrm{~m}, 8 \mathrm{H}, 2^{\prime}-, 3^{\prime}-, 6^{\prime}-, 7^{\prime}-\mathrm{H}\right), 7.29\left(\mathrm{~d}, J_{1,2}=15.79 \mathrm{~Hz}, 2 \mathrm{H}\right.$, 
$1-\mathrm{H}), 6.58\left(\mathrm{dd}, J_{2,3}=10.37 \mathrm{~Hz}, 2 \mathrm{H}, 2-\mathrm{H}\right), 6.41\left(\mathrm{dd}, J_{3,4}=15.30 \mathrm{~Hz}\right.$, 2H, 3-H), 5.80 (d, $2 \mathrm{H}, 4-\mathrm{H}), 1.72$ (s, $12 \mathrm{H}, \mathrm{CH}_{2}$ ).

$\mathrm{C}_{44} \mathrm{H}_{38}(566.8)$ Ber. C $93.24 \mathrm{H} 6.76$ Gef. C $92.86 \mathrm{H} 6.54$

Darstellung von (3-\{4-[4-(9-Anthryl)-1,3-butadien-1-yl]bicyclo[2.2.2]oct-1-yl\}-2-propen-1-yl)triphenylphosphoniumbromid (7)

1) 1-[4-(9-Anthryl)-1,3-butadien-1-yl]-4-[2-(hydroxymethyl)ethen-1-yl]bicyclo[2.2.2 Joctan: $\mathrm{Zu} 0.20 \mathrm{~g}(0.50 \mathrm{mmol}) 5$ in $70 \mathrm{ml}$ 1,2-Dichlorethan $/ 25 \mathrm{ml}$ Methanol werden bei Raumtemp. $0.80 \mathrm{~g}$ (21.1 mmol) aus Diethylenglycol-dimethylether (Diglyme) umkristallisiertes $\mathrm{NaBH}_{4}$ in $25 \mathrm{ml}$ Methanol unter Rühren getropft. Nach Beendigung der Reaktion (dünnschichtchromatographisch ermittelt) gießt man auf Wasser, trennt die organische Phase ab und extrahiert die wäßrige Phase dreimal mit Dichlormethan. Die vereinigten organischen Phasen werden getrocknet, i.Vak. eingeengt, und der Rückstand wird an Kieselgel mit Dichlormethan chromatographiert. Der Feststoff wird aus Toluol $/ n$-Hexan umkristallisiert; Ausb. $0.19 \mathrm{~g} \mathrm{(96 \% ),} \mathrm{Schmp.} 112^{\circ} \mathrm{C}$. $-(E)$-Isomere: ${ }^{1} \mathrm{H}-\mathrm{NMR}$ $\left(\mathrm{CDCl}_{3}\right): \delta=8.35\left(\mathrm{~s}, 1 \mathrm{H}, 10^{\prime}-\mathrm{H}\right), 8.32-8.28\left(\mathrm{~m}, 2 \mathrm{H}, 1^{\prime}-, 8^{\prime}-\mathrm{H}\right)$, $8.0-7.96\left(\mathrm{~m}, 2 \mathrm{H}, 4^{\prime}-, 5^{\prime}-\mathrm{H}\right), 7.48-7.42\left(\mathrm{~m}, 4 \mathrm{H}, 2^{\prime}-, 3^{\prime}-, 6^{\prime}-, 7^{\prime}-\mathrm{H}\right)$, $7.27\left(\mathrm{~d}, J_{1,2}=15.34 \mathrm{~Hz}, 1 \mathrm{H}, 1-\mathrm{H}\right), 6.55\left(\mathrm{dd}, J_{2.3}=10.34 \mathrm{~Hz}, 1 \mathrm{H}\right.$, $2-\mathrm{H}), 6.38\left(\mathrm{dd}, J_{3,4}=15.22 \mathrm{~Hz}, 1 \mathrm{H}, 3-\mathrm{H}\right), 5.76(\mathrm{~d}, 1 \mathrm{H}, 4-\mathrm{H}), 5.64$ (d, $\left.J_{2^{\prime \prime}, 1^{\prime \prime}}=15.90 \mathrm{~Hz}, 1 \mathrm{H}, 2^{\prime \prime}-\mathrm{H}\right), 5.51\left(\mathrm{dt}, J_{1^{\prime \prime}, \mathrm{CH}_{2}}=5.54 \mathrm{~Hz}, 1 \mathrm{H}, 1^{\prime \prime}-\right.$ H), $4.13-4.09\left(\mathrm{~m}, 2 \mathrm{H}, \mathrm{CH}_{2} \mathrm{OH}\right), 1.63-1.54\left(\mathrm{~m}, 12 \mathrm{H}, \mathrm{CH}_{2}\right)$.

\section{$\mathrm{C}_{29} \mathrm{H}_{30} \mathrm{O}$ (394.6) Ber. C 88.28 H 7.66 Gef. C 88.23 H 7.78}

2) $\mathrm{Zu} 0.10 \mathrm{~g}(0.25 \mathrm{mmol})$ 1-[4-(9-Anthryl)-1,3-butadien-1-yl]-4[2-(hydroxymethyl)ethen-1-yl]bicyclo[2.2.2]octan in $80 \mathrm{ml} \mathrm{Di-}$ chlormethan werden bei $0^{\circ} \mathrm{C} 0.14 \mathrm{~g}(0.51 \mathrm{mmol})$ Phosphortribromid in $10 \mathrm{ml}$ Dichlormethan getropft. Man läßt auf Raumtemp. erwärmen, rührt $3 \mathrm{~h}$ nach, gießt auf Eis/Wasser, extrahiert dreimal mit Dichlormethan, trocknet die vereinigten Extrakte und engt i.Vak. ein. Den Rückstand löst man in $50 \mathrm{ml}$ Toluol, gibt $1.00 \mathrm{~g}$ ( $3.81 \mathrm{mmol}$ ) Triphenylphosphan zu und erhitzt $2 \mathrm{~h}$ unter Rückfluß. Das erhaltene, in der Kälte auskristallisierte Öl wird abfiltriert und zweimal aus Diethylether/Dichlormethan umkristallisiert; Ausb. $0.10 \mathrm{~g}(55 \%) 7$, Schmp. $>270^{\circ} \mathrm{C}$ (Zers.). $-(E)$-Isomere: ${ }^{1} \mathrm{H}-\mathrm{NMR}$ $\left(\mathrm{CDCl}_{3}\right): \delta=8.35\left(\mathrm{~s}, 1 \mathrm{H}, 10^{\prime}-\mathrm{H}\right), 8.30-8.21\left(\mathrm{~m}, 2 \mathrm{H}, 1^{\prime}-, 8^{\prime}-\mathrm{H}\right)$, $8.0-7.93\left(\mathrm{~m}, 2 \mathrm{H}, 4^{\prime}-, 5^{\prime}-\mathrm{H}\right), 7.90-7.62\left(\mathrm{~m}, 15 \mathrm{H}, \mathrm{H}_{\text {Phenyl }}\right), 7.47-7.41$ (m, 4H, 2'-, $\left.3^{\prime}-, 6^{\prime}-, 7^{\prime}-\mathrm{H}\right), 7.25\left(\mathrm{~d}, J_{1,2}=15.57 \mathrm{~Hz}, 1 \mathrm{H}, 1-\mathrm{H}\right), 6.52$ $\left(\mathrm{dd}, J_{2,3}=10.45 \mathrm{~Hz}, 1 \mathrm{H}, 2-\mathrm{H}\right), 6.32\left(\mathrm{dd}, J_{3,4}=15.23 \mathrm{~Hz}, 1 \mathrm{H}, 3-\right.$ H), $5.72\left(\mathrm{~d}, J_{2^{\prime \prime}, 1^{\prime \prime}}=15.66 \mathrm{~Hz}, 1 \mathrm{H}, 2^{\prime \prime}-\mathrm{H}\right), 5.69(\mathrm{~d}, 1 \mathrm{H}, 4-\mathrm{H}), 5.16(\mathrm{dt}$, $\left.J_{1^{\prime \prime}, \mathrm{CH}_{2}}=7.42 \mathrm{~Hz}, 1 \mathrm{H}, 1^{\prime \prime}-\mathrm{H}\right), 4.73\left(\mathrm{dd}, J_{\mathrm{CH}_{2}, \mathrm{P}}=15.0 \mathrm{~Hz}, 2 \mathrm{H}, \mathrm{CH}_{2} \mathrm{P}\right)$, $1.58-1.35\left(\mathrm{~m}, 12 \mathrm{H}, \mathrm{CH}_{2}\right)$. - MS (EI, $\left.70 \mathrm{eV}\right): m / z(\%)(719.7): 378$ (3) $\left[\mathrm{M}^{+\cdot}-\mathrm{Ph}_{3} \mathrm{P}^{+\cdot}-\mathrm{Br}^{\cdot-}\right], 338(4)\left[\mathrm{M}^{+\cdot}-\mathrm{C}_{3} \mathrm{H}_{4}^{+\cdot}-\mathrm{Ph}_{3} \mathrm{P}^{+\cdot}-\right.$ $\left.\mathrm{Br}^{\cdot-}\right], 262(31)\left[\mathrm{Ph}_{3} \mathrm{P}^{+\cdot}\right], 229$ (11) $\left[\mathrm{C}_{18} \mathrm{H}_{12}^{+*}\right], 192(38)\left[\mathrm{C}_{15} \mathrm{H}_{10}^{+\cdot}+\right.$ 1], 191 (51) $\left[\mathrm{C}_{15} \mathrm{H}_{10}^{+}\right], 178(100)\left[\mathrm{C}_{14} \mathrm{H}_{10}^{+*}\right.$ (Anthracen) $], 108$ (15) [ $\mathrm{C}_{8} \mathrm{H}_{12}$ (Bicyclus)], 79 (11) $\left[\mathrm{Br}^{-\cdot}\right]$.

\section{2-Formyl-5,10,15,20-tetraphenyl-21H,23H-porphyrin: Nach Lit. ${ }^{[16 a]}$}

2-Methyl-5-[2-(5,10,15,20-tetraphenyl-21H,23H-porphyrinyl)]2,4-pentadienal (8): In einen Dreihalskolben mit Rückflußkühler, Tropftrichter und Gasableitungsrohr gibt man bei Raumtemp. zu $4.00 \mathrm{ml}$ (4.00 mmol) einer $1 \mathrm{M}$ Lösung von (4,4-Dimethoxy-3-methyl-2-butenyl)triphenylphosphoniumchlorid in Methanol $1.00 \mathrm{ml}$ (4.00 mmol) einer frisch hergestellten $4 \mathrm{M}$ Lösung von Natriummethanolat in Methanol und rührt $22 \mathrm{~h}$. Dann werden $0.50 \mathrm{~g}(0.77$ mmol) 2-Formyl-5,10,15,20-tetraphenyl-21 $H, 23 H$-porphyrin in $50 \mathrm{ml}$ 1,2-Dichlorethan zugetropft. Nach Beendigung der Reaktion (dünnschichtchromatographisch verfolgt) gießt man auf Wasser, extrahiert dreimal mit Dichlormethan, trocknet die vereinigten Extrakte, engt ein und chromatographiert den Rückstand an Kieselgel mit Dichlormethan. Der Feststoff wird aus Dichlormethan/Hexan umkristallisiert und i. Vak. getrocknet; Ausb. $0.30 \mathrm{~g}$ (55\%), Schmp. $>270^{\circ} \mathrm{C} .-(E)$-Isomere: ${ }^{1} \mathrm{H}-\mathrm{NMR}\left(\mathrm{CDCl}_{3}\right): \delta=9.47(\mathrm{~s}, 1 \mathrm{H}$, CHO), 8.98 (s, $\left.1 \mathrm{H}, 3^{\prime \prime \prime}-\mathrm{H}\right), 8.82\left(\mathrm{~d}, J_{18^{\prime \prime}, 17^{\prime \prime \prime}}=4.87 \mathrm{~Hz}, 1 \mathrm{H}, 18^{\prime \prime \prime}-\mathrm{H}\right.$ ), $8.80-8.70\left(\mathrm{~m}, 5 \mathrm{H}, 7^{\prime \prime \prime}-, 8^{\prime \prime \prime}-, 12^{\prime \prime \prime}-, 13^{\prime \prime \prime}-, 17^{\prime \prime \prime}-\mathrm{H}\right), 8.24-8.10(\mathrm{~m}, 8 \mathrm{H}$, $\left.o-\mathrm{H}_{\text {Phenyl }}\right), 7.86-7.71\left(\mathrm{~m}, 12 \mathrm{H}, m-, p-\mathrm{H}_{\text {Phenyl }}\right), 7.34\left(\mathrm{dd}, J_{4,5}=\right.$ $\left.15.28 \mathrm{~Hz}, J_{4,3}=11.41 \mathrm{~Hz}, 1 \mathrm{H}, 4-\mathrm{H}\right), 6.60(\mathrm{~d}, 1 \mathrm{H}, 3-\mathrm{H}), 6.58(\mathrm{~d}, 1 \mathrm{H}$, 5-H), 1.98 (s, 3 H, $\left.\mathrm{CH}_{3}\right),-2.59$ (s, 2H, NH).

$\mathrm{C}_{50} \mathrm{H}_{36} \mathrm{~N}_{4} \mathrm{O}(708.9) \quad$ Ber. C 84.72 H 5.12 N 7.90

Gef. C 84.01 H 5.19 N 7.79

2,7-Dimethyl-9-[2-(5,10,15,20-tetraphenyl-21H,23H-porphyrinyl)]2,4,6,8-nonatetraenal (9): Nach Lit. ${ }^{[7]}$.

Kopplung der TPP-Polyenale 8 und 9 mit 7: In einen Dreihalskolben mit Rückflußkühler, Tropftrichter und Sicapent-Trockenrohr spritzt man bei Raumtemp. unter $\mathrm{N}_{2}$ zu 7 in $20 \mathrm{ml}$ THF eine Lösung von BuLi in Hexan, rührt 30 min und setzt zur Zersetzung des BuLi-Überschusses $1 \mathrm{ml}$ Dichlormethan zu. Dann wird 8 bzw. 9 in 1,2-Dichlorethan zugetropft und die Mischung die angegebene Zeit gerührt. Das Reaktionsgemisch gießt man auf Wasser, trennt die organische Phase ab und extrahiert die wäßrige mit Dichlormethan. Die vereinigten organischen Phasen trocknet man mit Magnesiumsulfat, engt ein und chromatographiert den Rückstand an Kieselgel.

1) 4-[4-(9-Anthryl)-1,3-butadien-1-yl]-1-[5-methyl-8-[2$(5,10,15,20$-tetraphenyl-21H,23H-porphyrinyl) $]-1,3,5,7$-octatetraen1 -ylJbicyclo[2.2.2 Joctan (10): Aus $88 \mathrm{mg}(0.13 \mathrm{mmol}) 7,0.8 \mathrm{ml}(1.28$ mmol) einer $1.6 \mathrm{M}$ Lösung von BuLi, $90 \mathrm{mg}(0.13 \mathrm{mmol}) \mathrm{8}$, durch 23stdg. Rühren und Chromatographie mit Dichlormethan/Ethylacetat $(9: 1)$; Ausb. $72 \mathrm{mg}(53 \%)$. Die (Z)-Isomeren werden mittels MPLC an Kieselgel mit Hexan/Ethylacetat (94:6) und einem Fluß von $50 \mathrm{ml} / \mathrm{min}$ (Detektion bei $\lambda=257$ bzw. $425 \mathrm{~nm}$ ) abgetrennt, und das $(E)$-Isomer wird aus Dichlormethan/Hexan umkristallisiert; Ausb. $12 \mathrm{mg}(9 \%)$, Schmp. $>270^{\circ} \mathrm{C}$. $-{ }^{1} \mathrm{H}-\mathrm{NMR}\left(\mathrm{CDCl}_{3}\right): \delta=$ 8.91 (s, $\left.1 \mathrm{H}, 3^{\prime \prime \prime}-\mathrm{H}\right), 8.80-8.69\left(\mathrm{~m}, 6 \mathrm{H}, 7^{\prime \prime \prime}-, 8^{\prime \prime \prime}-, 12^{\prime \prime \prime}-, 13^{\prime \prime \prime}-, 17^{\prime \prime \prime}-\right.$, $\left.18^{\prime \prime \prime}-\mathrm{H}\right), 8.26\left(\mathrm{~s}, 1 \mathrm{H}, 10^{\prime}-\mathrm{H}\right), 8.26-8.23\left(\mathrm{~m}, 2 \mathrm{H}, 1^{\prime}-, 8^{\prime}-\mathrm{H}\right), 8.19-8.07$ $\left(\mathrm{m}, 8 \mathrm{H}, o-\mathrm{H}_{\text {Phenyl }}\right), 7.90-7.80\left(\mathrm{~m}, 2 \mathrm{H}, 4^{\prime}-, 5^{\prime}-\mathrm{H}\right), 7.77-7.71(\mathrm{~m}, 12 \mathrm{H}$, $\left.m-, p-\mathrm{H}_{\text {Phenyl }}\right), 7.40-7.39\left(\mathrm{~m}, 4 \mathrm{H}, 2^{\prime}-, 3^{\prime}-, 6^{\prime}-, 7^{\prime}-\mathrm{H}\right), 7.33$ (d, $J_{1,2}=$ $15.04 \mathrm{~Hz}, 1 \mathrm{H}, 1-\mathrm{H}), 7.28\left(\mathrm{~d}, J_{1^{\prime \prime}, 2^{\prime \prime}}=15.14 \mathrm{~Hz}, 1 \mathrm{H}, 1^{\prime \prime}-\mathrm{H}\right), 6.55(\mathrm{dd}$, $\left.J_{2,3}=10.38 \mathrm{~Hz}, 1 \mathrm{H}, 2-\mathrm{H}\right), 6.35\left(\mathrm{dd}, J_{2^{n}, 3^{\prime \prime}}=10.26 \mathrm{~Hz}, 1 \mathrm{H}, 2^{\prime \prime}-\mathrm{H}\right)$, $6.34\left(\mathrm{dd}, J_{3,4}=15.34 \mathrm{~Hz}, 1 \mathrm{H}, 3-\mathrm{H}\right), 6.25\left(\mathrm{dd}, J_{7^{*}, 8^{\prime \prime}}=15.90 \mathrm{~Hz}\right.$, $\left.J_{7^{\prime \prime}, 6^{\prime \prime}}=10.54 \mathrm{~Hz}, 1 \mathrm{H}, 7^{\prime \prime}-\mathrm{H}\right), 6.16\left(\mathrm{~d}, J_{5^{\prime \prime}, 6^{\prime \prime}}=14.84 \mathrm{~Hz}, 1 \mathrm{H}, 5^{\prime \prime}-\mathrm{H}\right)$, $6.10\left(\mathrm{dd}, 1 \mathrm{H}, 6^{\prime \prime}-\mathrm{H}\right), 5.98\left(\mathrm{~d}, 1 \mathrm{H}, 3^{\prime \prime}-\mathrm{H}\right), 5.70(\mathrm{~d}, 1 \mathrm{H}, 4-\mathrm{H}), 5.69$ (d, $\left.1 \mathrm{H}, 8^{\prime \prime}-\mathrm{H}\right), 2.02\left(\mathrm{~s}, 3 \mathrm{H}, \mathrm{CH}_{3}\right), 1.67\left(\mathrm{~s}, 12 \mathrm{H}, \mathrm{CH}_{2}\right),-2.56(\mathrm{~s}, 2 \mathrm{H}$, NH). - MS (EI, $70 \mathrm{eV}): m / z(\%)(1069.4): 156(100)\left[\mathrm{C}_{12} \mathrm{H}_{14}^{+*}\right], 178$ (90) $\left[\mathrm{C}_{14} \mathrm{H}_{10}^{+} \cdot\right], 191$ (43) $\left[\mathrm{C}_{15} \mathrm{H}_{10}^{+}{ }^{*}\right], 192$ (45) $\left[\mathrm{C}_{15} \mathrm{H}_{11}^{+}\right], 217$ (11) $\left[\mathrm{C}_{17} \mathrm{H}_{12}^{+*}\right], 230(4)\left[\mathrm{C}_{18} \mathrm{H}_{13}^{+}{ }^{*}\right], 614$ (7) [TPP], $1068(6)\left[\mathrm{M}^{+\cdot}\right]$. $\mathrm{C}_{79} \mathrm{H}_{64} \mathrm{~N}_{4}$ Ber. 1068.5121 Gef. 1068.5119 (MS)

2) 4-[4-(9-Anthryl)-1,3-butadien-1-yl]-1-[5,10,-dimethyl-12-[2$(5,10,15,20$-tetraphenyl-21H,23H-porphyrinyl) $]-1,3,5,7,9,11$-dodecahexaen-1-yljbicyclo/2.2.2 Joctan (11): Aus $130 \mathrm{mg}(0.18 \mathrm{mmol}) 7$, $1.00 \mathrm{ml}(1.60 \mathrm{mmol})$ einer $1.6 \mathrm{M}$ Lösung von BuLi, $180 \mathrm{mg}(0.23$ mmol) 9, durch 18stdg. Rühren und Chromatographie mit Petrolether/Ethylacetat (8:2); Ausb. $70 \mathrm{mg}$ (31\%). Die (Z)-Isomeren werden mittels MPLC an Nucleosil $7 \mathrm{NO}_{2}$ mit Hexan/Dichlormethan (65:35) und einem Fluß von $50 \mathrm{ml} / \mathrm{min}$ (Detektion bei $\lambda=257$ bzw. $425 \mathrm{~nm}$ ) abgetrennt, und das (E)-Isomere wird aus Ethylacetat/ Hexan umkristallisiert; Ausb. $10 \mathrm{mg}(4 \%)$, Schmp. $>270^{\circ} \mathrm{C}$. $-{ }^{1} \mathrm{H}$ NMR $\left(\mathrm{CDCl}_{3}\right): \delta=8.91\left(\mathrm{~s}, 1 \mathrm{H}, 3^{\prime \prime \prime}-\mathrm{H}\right), 8.83-8.71\left(\mathrm{~m}, 5 \mathrm{H}, 7^{\prime \prime \prime}-, 8^{\prime \prime \prime}-\right.$, $\left.12^{\prime \prime \prime}-, 13^{\prime \prime \prime}-, 17^{\prime \prime \prime}-\mathrm{H}\right), 8.64$ (d, $\left.J_{18^{\prime \prime \prime}, 17^{\prime \prime}}=4.55 \mathrm{~Hz}, 1 \mathrm{H}, 18^{\prime \prime \prime}-\mathrm{H}\right), 8.35$ (s, $\left.1 \mathrm{H}, 10^{\prime}-\mathrm{H}\right), 8.33-8.27\left(\mathrm{~m}, 2 \mathrm{H}, 1^{\prime}-, 8^{\prime}-\mathrm{H}\right), 8.24-8.09(\mathrm{~m}, 8 \mathrm{H}, 0-$ $\left.\mathrm{H}_{\text {Phenyl }}\right), 8.01-7.96\left(\mathrm{~m}, 2 \mathrm{H}, 4^{\prime}-, 5^{\prime}-\mathrm{H}\right), 7.81-7.69(\mathrm{~m}, 12 \mathrm{H}, m-, p-$ $\left.\mathrm{H}_{\text {Phenyl }}\right), 7.52-7.49\left(\mathrm{~m}, 4 \mathrm{H}, 2^{\prime}-, 3^{\prime}-, 6^{\prime}-, 7^{\prime}-\mathrm{H}\right), 7.27$ (d, $J_{1,2}=$ $16.21 \mathrm{~Hz}, 1 \mathrm{H}, 1-\mathrm{H}), 7.12\left(\mathrm{~d}, J_{1^{\prime \prime}, 2^{\prime \prime}}=15.46 \mathrm{~Hz}, 1 \mathrm{H}, 1^{\prime \prime}-\mathrm{H}\right), 6.65(\mathrm{dd}$, $\left.J_{2,3}=10.71 \mathrm{~Hz}, 1 \mathrm{H}, 2-\mathrm{H}\right), 6.56\left(\mathrm{dd}, J_{3,4}=15.30 \mathrm{~Hz}, 1 \mathrm{H}, 3-\mathrm{H}\right), 6.49$ 
(d, $\left.1 \mathrm{H}, 2^{\prime \prime}-\mathrm{H}\right), 6.42\left(\mathrm{~d}, J_{4^{\prime \prime}, 5^{*}}=10.09 \mathrm{~Hz}, 1 \mathrm{H}, 4^{\prime \prime}-\mathrm{H}\right), 6.41$ (d, $J_{7^{*}, 6^{*}}=$ $\left.10.04 \mathrm{~Hz}, 1 \mathrm{H}, 7^{\prime \prime}-\mathrm{H}\right), 6.35$ (dd, $\left.J_{5^{\prime \prime}, 6^{\prime \prime}}=14.23 \mathrm{~Hz}, 1 \mathrm{H}, 5^{\prime \prime}-\mathrm{H}\right)$, $6.35-6.21\left(\mathrm{~m}, 2 \mathrm{H}, 6^{\prime \prime}-, 9^{\prime \prime}-\mathrm{H}\right), 6.10\left(\mathrm{dd}, J_{10^{\prime \prime}, 9^{\prime \prime}}=15.15 \mathrm{~Hz}, J_{10^{\prime \prime}, 11^{\prime \prime}}=\right.$ $10.36 \mathrm{~Hz}, 1 \mathrm{H}, 10^{\prime \prime}-\mathrm{H}$ ), 6.05 (dd, $J_{11^{\prime \prime}, 12^{\prime \prime}}=15.26 \mathrm{~Hz}, 1 \mathrm{H}, 11^{\prime \prime}-\mathrm{H}$ ), 5.74 (d, $1 \mathrm{H}, 4-\mathrm{H}), 5.73$ (d, $\left.1 \mathrm{H}, 12^{\prime \prime}-\mathrm{H}\right), 2.05$ (s, 3H, 3"- $\mathrm{CH}_{3}$ ), 1.94 (s, 3H, $\left.8^{\prime \prime}-\mathrm{CH}_{3}\right), 1.68-1.51\left(\mathrm{~m}, 12 \mathrm{H}, \mathrm{CH}_{2}\right),-2.55(\mathrm{~s}, 2 \mathrm{H}, \mathrm{NH})$.

$$
\begin{array}{lllll}
\mathrm{C}_{84} \mathrm{H}_{70} \mathrm{~N}_{4} \text { (1135.5) } & \text { Ber. C } 88.85 \text { H } 6.21 \text { N } 4.93 \\
& \text { Gef. C } 87.31 \text { H } 6.32 \text { N } 4.80
\end{array}
$$

5-(9-Anthryl)-2-methyl-2,4-pentadienal (12): Wie für 8 beschrieben, aus $5.00 \mathrm{~g}(24.2 \mathrm{mmol}) 9-$ Formylanthracen in $50 \mathrm{ml} 1,2-\mathrm{Di}$ chlorethan, $50.0 \mathrm{ml}$ (50.0 mmol) einer $1 \mathrm{M}$ Lösung von (4,4-Dimethoxy-3-methyl-2-butenyl)triphenylphosphoniumchlorid in Methanol, $12.5 \mathrm{ml}$ ( $50.0 \mathrm{mmol})$ einer $4 \mathrm{M}$ Lösung von Natriummethanolat in Methanol; Ausb. $3.00 \mathrm{~g}(45 \%)$, Schmp. $125^{\circ} \mathrm{C}$ (Ethanol) (Lit. ${ }^{[19]}$ $\left.124-125^{\circ} \mathrm{C}\right) .-(E)$-Isomere: ${ }^{1} \mathrm{H}-\mathrm{NMR}\left(\mathrm{CDCl}_{3}\right): \delta=9.63(\mathrm{~s}, 1 \mathrm{H}$, CHO), 8.42 (s, 1 H, 10'-H), 8.26-8.23 (m, 2H, $\left.1^{\prime}-, 8^{\prime}-\mathrm{H}\right), 8.02-7.99$ $\left(\mathrm{m}, 2 \mathrm{H}, 4^{\prime}-, 5^{\prime}-\mathrm{H}\right), 7.88\left(\mathrm{~d}, J_{5,4}=15.47 \mathrm{~Hz}, 1 \mathrm{H}, 5-\mathrm{H}\right), 7.53-7.46$ $\left(\mathrm{m}, 4 \mathrm{H}, 2^{\prime}-, 3^{\prime}-, 6^{\prime}-, 7^{\prime}-\mathrm{H}\right), 7.38\left(\mathrm{~d}, J_{3,4}=11.35 \mathrm{~Hz}, J_{3, \mathrm{CH}_{3}}=1.67 \mathrm{~Hz}\right.$, $1 \mathrm{H}, 3-\mathrm{H}), 7.15(\mathrm{dd}, 1 \mathrm{H}, 4-\mathrm{H}), 1.91\left(\mathrm{~d}, 3 \mathrm{H}, 2-\mathrm{CH}_{3}\right)$.

$\mathrm{C}_{20} \mathrm{H}_{16} \mathrm{O}$ (272.3) Ber. C 88.20 H 5.92 Gef. C 88.25 H 5.96

Darstellung von [5-(9-Anthryl)-2-methyl-2,4-pentadien-1-yl]triphenylphosphoniumbromid (13)

1) 5-(9-Anthryl)-2-methyl-2,4-pentadienol: Analog 1) bei der Darstellung von 7 , aus $3.00 \mathrm{~g}$ (11.0 mmol) 12 in $80 \mathrm{ml}$ Dichlormethan/

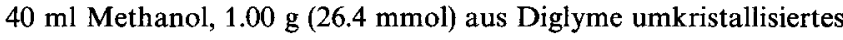
$\mathrm{NaBH}_{4}$ in $20 \mathrm{ml}$ Methanol; Ausb. $2.50 \mathrm{~g}(83 \%)$, Schmp. $87^{\circ} \mathrm{C}$ (Dichlormethan/Hexan). - (E)-Isomere: ${ }^{1} \mathrm{H}-\mathrm{NMR}\left(\mathrm{CDCl}_{3}\right): \delta=8.36$ (s, $\left.1 \mathrm{H}, 10^{\prime}-\mathrm{H}\right), 8.33-8.25\left(\mathrm{~m}, 2 \mathrm{H}, 1^{\prime}-, 8^{\prime}-\mathrm{H}\right), 8.02-7.94(\mathrm{~m}, 2 \mathrm{H}$, $\left.4^{\prime}-, 5^{\prime}-\mathrm{H}\right), 7.54-7.41\left(\mathrm{~m}, 4 \mathrm{H}, 2^{\prime}-, 3^{\prime}-, 6^{\prime}-, 7^{\prime}-\mathrm{H}\right), 7.36\left(\mathrm{~d}, J_{5,4}=\right.$ $15.71 \mathrm{~Hz}, 1 \mathrm{H}, 5-\mathrm{H}), 6.86\left(\mathrm{dd}, J_{4,3}=11.0 \mathrm{~Hz}, 1 \mathrm{H}, 4-\mathrm{H}\right), 6.56(\mathrm{dd}$, $\left.J_{3, \mathrm{CH}_{3}}=0.66 \mathrm{~Hz}, 1 \mathrm{H}, 3-\mathrm{H}\right), 4.22-4.21\left(\mathrm{~m}, 2 \mathrm{H}, \mathrm{CH}_{2} \mathrm{OH}\right), 1.84(\mathrm{~d}$, $3 \mathrm{H}, 2-\mathrm{CH}_{3}$ ).

\section{$\mathrm{C}_{20} \mathrm{H}_{18} \mathrm{O}(274.4)$ Ber. C 87.56 H 6.61 Gef. C 87.66 H 6.68}

2) Analog 2) bei der Darstellung von 7, jedoch 1stdg. Rühren nach Zugabe von Phosphortribromid, 2stdg. Erhitzen auf $60^{\circ} \mathrm{C}$ nach Zugabe von Triphenylphosphan, Umkristallisation aus Ethanol und Chromatographie an Kieselgel mit Methanol/Dichlormethan (60:40), aus $2.50 \mathrm{~g}(9.11 \mathrm{mmol})$ 5-(9-Anthryl)-2-methyl-2,4pentadienol in $50 \mathrm{ml}$ Dichlormethan, $12.3 \mathrm{~g}(45.6 \mathrm{mmol})$ Phosphortribromid in $10 \mathrm{ml}$ Dichlormethan, $4.00 \mathrm{~g}(15.3 \mathrm{mmol})$ Triphenylphosphan in $100 \mathrm{ml}$ Toluol; Ausb. $1.70 \mathrm{~g} \mathrm{(31 \% )} 13$ als $(E) /$ (Z)-Gemisch (78:22), Schmp. $147-148^{\circ} \mathrm{C}$. $-(E)$-Isomere: ${ }^{1} \mathrm{H}$ NMR $\left(\mathrm{CDCl}_{3}\right): \delta=8.31\left(\mathrm{~s}, 1 \mathrm{H}, 10^{\prime}-\mathrm{H}\right), 8.11-8.07\left(\mathrm{~m}, 2 \mathrm{H}, 1^{\prime}-, 8^{\prime}-\right.$ H), $7.95-7.68$ (m, 17H, 4'-, 5'-H, $\left.\mathrm{H}_{\text {Phenyl }}\right), 7.44-7.38$ (m, 4H, 2'-, $\left.3^{\prime}-, 6^{\prime}-, 7^{\prime}-\mathrm{H}\right), 7.13\left(\mathrm{~d}, J_{5,4}=15.62 \mathrm{~Hz}, 1 \mathrm{H}, 5-\mathrm{H}\right), 6.63\left(\mathrm{dd}, J_{4,3}=\right.$ $10.90 \mathrm{~Hz}, 1 \mathrm{H}, 4-\mathrm{H}), 6.35(\mathrm{~d}, 1 \mathrm{H}, 3-\mathrm{H}), 4.89\left(\mathrm{~d}, J_{\mathrm{CH}_{2}, \mathrm{P}}=15.51 \mathrm{~Hz}\right.$, $\left.2 \mathrm{H}, \mathrm{CH}_{2} \mathrm{P}\right), 1.67-1.66\left(\mathrm{~m}, 3 \mathrm{H}, \mathrm{CH}_{3}\right)$ - MS (EI, $\left.70 \mathrm{eV}\right): \mathrm{m} / z(\%)$ (599.5): 516 (1) $\left[\mathrm{M}^{+\cdot}-\mathrm{Br}^{-*}\right], 262(100)\left[\mathrm{Ph}_{3} \mathrm{P}^{+\cdot}\right], 258$ (98) $\left[\mathrm{M}^{+\cdot}-\mathrm{Ph}_{3} \mathrm{P}^{+\cdot} / \mathrm{Br}^{-\cdot}\right], 229(4)\left[\mathrm{C}_{19} \mathrm{H}_{15}^{+}\right], 215(78)\left[\mathrm{C}_{17} \mathrm{H}_{12}^{+}\right], 203$ (25) $\left[\mathrm{C}_{16} \mathrm{H}_{11}^{+*}\right], 202(49)\left[\mathrm{C}_{16} \mathrm{H}_{10}^{+*}\right], 191$ (27) $\left[\mathrm{C}_{15} \mathrm{H}_{10}^{+*}\right], 178$ (22) $\left[\mathrm{C}_{14} \mathrm{H}_{9}^{+\cdot}\right], 79(42)\left[\mathrm{Br}^{-\cdot}\right]$

Umsetzung von 13 mit 3: Wie für 5, 6 beschrieben, aus $1.20 \mathrm{~g}(5.50$ $\mathrm{mmol}) 3$ in $50 \mathrm{ml} \mathrm{1,2-Dichlorethan,} 3.00 \mathrm{~g}(5.00 \mathrm{mmol}) 13$ in $50 \mathrm{ml}$ THF, $6.00 \mathrm{ml}(4.80 \mathrm{mmol})$ einer $0.8 \mathrm{M}$ Lösung von BuLi in Hexan; Ausb. $0.36 \mathrm{~g}(15 \%) 15$, daneben $0.04 \mathrm{~g}(1.0 \%) 14$, Schmp. $124^{\circ} \mathrm{C}$ (Dichlormethan/Methanol). Die 4 Isomere von 15 werden mittels MPLC an Kieselgel mit Hexan/Ethylacetat $(94: 6)$ und einem Fluß von $50 \mathrm{ml} / \mathrm{min}$ (Detektion bei $\lambda=257 \mathrm{~nm}$ ) abgetrennt: Fraktion 1: Ausb. $5 \mathrm{mg}(0.2 \%)(3 Z, 5 Z)-15$, Schmp. $103^{\circ} \mathrm{C}$; Fraktion 2: Ausb. $105 \mathrm{mg}(4 \%)(3 Z)-15$, Schmp. $122-123^{\circ} \mathrm{C}$; Fraktion 3: Ausb. $250 \mathrm{mg}(10 \%)(E)$-Isomer von 15 , Schmp. $192-195^{\circ} \mathrm{C} .-{ }^{1} \mathrm{H}-\mathrm{NMR}$
$\left(\mathrm{CDCl}_{3}\right): \delta=9.50(\mathrm{~d}, 1 \mathrm{H}, \mathrm{CHO}), 8.36\left(\mathrm{~s}, 1 \mathrm{H}, 10^{\prime}-\mathrm{H}\right), 8.35-8.28(\mathrm{~m}$, $\left.2 \mathrm{H}, 1^{\prime}-, 8^{\prime}-\mathrm{H}\right), 8.02-7.96\left(\mathrm{~m}, 2 \mathrm{H}, 4^{\prime}-, 5^{\prime}-\mathrm{H}\right), 7.48-7.44(\mathrm{~m}, 4 \mathrm{H}$, $\left.2^{\prime}-, 3^{\prime}-, 6^{\prime}-, 7^{\prime}-\mathrm{H}\right), 7.42(\mathrm{~d}, 1 \mathrm{H}, 1-\mathrm{H}), 7.0\left(\mathrm{dd}, J_{2,1}=15.55 \mathrm{~Hz}, J_{2,3}=\right.$ $11.27 \mathrm{~Hz}, 1 \mathrm{H}, 2-\mathrm{H}), 6.72\left(\mathrm{~d}, J_{2^{\prime \prime}, 1^{\prime \prime}}=15.88 \mathrm{~Hz}, 1 \mathrm{H}, 2^{\prime \prime}-\mathrm{H}\right), 6.54(\mathrm{~d}$, $1 \mathrm{H}, 3-\mathrm{H}), 6.40\left(\mathrm{~d}, J_{5,6}=15.56 \mathrm{~Hz}, 1 \mathrm{H}, 5-\mathrm{H}\right), 6.32\left(\mathrm{dd}, J_{6,7}=\right.$ $10.09 \mathrm{~Hz}, 1 \mathrm{H}, 6-\mathrm{H}), 6.09\left(\mathrm{dd}, J_{7,8}=15.41 \mathrm{~Hz}, 1 \mathrm{H}, 7-\mathrm{H}\right), 6.0(\mathrm{dd}$, $\left.J_{1^{\prime \prime}, \mathrm{CHO}}=7.75 \mathrm{~Hz}, 1 \mathrm{H}, 1^{\prime \prime}-\mathrm{H}\right), 5.69(\mathrm{~d}, 1 \mathrm{H}, 8-\mathrm{H}), 1.93\left(\mathrm{~s}, 3 \mathrm{H}, \mathrm{CH}_{3}\right)$, $1.63\left(\mathrm{~s}, 12 \mathrm{H}, \mathrm{CH}_{2}\right)$.

\section{$\mathrm{C}_{34} \mathrm{H}_{34} \mathrm{O}$ (458.6) Ber. C 89.04 H 7.47 Gef. C 88.95 H 7.33}

$(E) /(Z)$-Isomere von $14:{ }^{1} \mathrm{H}-\mathrm{NMR}\left(\mathrm{CDCl}_{3}\right): \delta=8.37,8.36(2 \mathrm{~s}$, $\left.2 \mathrm{H}, 10^{\prime}-\mathrm{H}\right), 8.34-8.26\left(\mathrm{~m}, 4 \mathrm{H}, 1^{\prime}-, 8^{\prime}-\mathrm{H}\right), 8.0-7.93\left(\mathrm{~m}, 4 \mathrm{H}, 4^{\prime}-, 3^{\prime}-\right.$ H), $7.5-7.37\left(\mathrm{~m}, 8 \mathrm{H}, 2^{\prime}-, 3^{\prime}-, 6^{\prime}-, 7^{\prime}-\mathrm{H}\right), 7.06-6.94(\mathrm{~m}, 2 \mathrm{H}, 2-\mathrm{H})$, $6.6-6.52(\mathrm{~m}, 2 \mathrm{H}, 3-\mathrm{H}), 6.37-6.33(\mathrm{~m}, 2 \mathrm{H}, 5-\mathrm{H}), 6.13-5.9(\mathrm{~m}, 4 \mathrm{H}$, 6-, 7-H), 5.76-5.65 (m, 2H, 8-H), 2.11-1.92(m, 6H, $\left.\mathrm{CH}_{3}\right)$, $1.66-1.42\left(\mathrm{~m}, 12 \mathrm{H}, \mathrm{CH}_{2}\right)$.

(3-\{4-[8-(9-Anthryl)-5-methyl-1,3,5,7-octatetraen-1-yl]bicyclo[2.2.2]oct-1-yl\}-2-propen-1-yl) triphenylphosphoniumbromid (16)

1) 1-[8-(9-Anthryl)-5-methyl-1,3,5,7-octatetraen-1-yl]-4-[2-(hydroxymethyl)ethen-1-yl Jbicyclo(2.2.2 Joctan: Analog 1) bei der Darstellung von 7, jedoch Umkristallisation aus Hexan/Ethylacetat (98:2), aus $0.25 \mathrm{~g}(0.55 \mathrm{mmol}) 15 \mathrm{in} 100 \mathrm{ml}$ 1,2-Dichlorethan $/ 100 \mathrm{ml}$ Methanol, $1.00 \mathrm{~g}(26.4 \mathrm{mmol}) \mathrm{NaBH}_{4}$; Ausg. $0.24 \mathrm{~g}$ (95\%), Schmp. $109^{\circ} \mathrm{C}$. $-{ }^{1} \mathrm{H}-\mathrm{NMR}\left(\mathrm{CDCl}_{3}\right): \delta=8.34\left(\mathrm{~s}, 1 \mathrm{H}, 10^{\prime}-\mathrm{H}\right), 8.33-8.28$ $\left(\mathrm{m}, 2 \mathrm{H}, 1^{\prime}-, 8^{\prime}-\mathrm{H}\right), 8.0-7.95\left(\mathrm{~m}, 2 \mathrm{H}, 4^{\prime}-, 5^{\prime}-\mathrm{H}\right), 7.53-7.43(\mathrm{~m}, 4 \mathrm{H}$, $\left.2^{\prime}-, 3^{\prime}-, 6^{\prime}-, 7^{\prime}-\mathrm{H}\right), 7.41(\mathrm{~d}, 1 \mathrm{H}, 1-\mathrm{H}), 7.0\left(\mathrm{dd}, J_{2,1}=15.58 \mathrm{~Hz}, J_{2,3}=\right.$ $11.38 \mathrm{~Hz}, 1 \mathrm{H}, 2-\mathrm{H}), 6.52(\mathrm{~d}, 1 \mathrm{H}, 3-\mathrm{H}), 6.42-6.27(\mathrm{~m}, 2 \mathrm{H}, 5-, 6-\mathrm{H})$, $6.07\left(\mathrm{dd}, J_{7,8}=15.48 \mathrm{~Hz}, J_{7,6}=9.64 \mathrm{~Hz}, 1 \mathrm{H}, 7-\mathrm{H}\right), 5.7(\mathrm{~d}, 1 \mathrm{H}, 8-$ H), $5.6\left(\mathrm{~d}, J_{2^{\prime \prime}, 1^{\prime \prime}}=15.82 \mathrm{~Hz}, 1 \mathrm{H}, 2^{\prime \prime}-\mathrm{H}\right), 5.48\left(\mathrm{dt}, J_{1^{\prime \prime}, \mathrm{CH}_{2}}=5.73 \mathrm{~Hz}\right.$, $\left.1 \mathrm{H}, 1^{\prime \prime}-\mathrm{H}\right), 4.08\left(\mathrm{~s}, 2 \mathrm{H}, \mathrm{CH}_{2} \mathrm{OH}\right), 1.92\left(\mathrm{~s}, 3 \mathrm{H}, 4-\mathrm{CH}_{3}\right), 1.6-1.49(\mathrm{~m}$, $12 \mathrm{H}, \mathrm{CH}_{2}$ ). $-\mathrm{MS}(\mathrm{EI}, 70 \mathrm{eV}): m / z(\%)(460.7): 461(36)\left[\mathrm{M}^{+}{ }^{+}+\right.$ 1], 460 (100) $\left[\mathrm{M}^{+\cdot}\right.$, Basispeak], 281 (53) $\left[\mathrm{C}_{22} \mathrm{H}_{17}^{+}\right], 267$ (41) $\left[\mathrm{C}_{21} \mathrm{H}_{15}^{+}{ }^{*}\right], 216(12)\left[\mathrm{C}_{17} \mathrm{H}_{12}^{+*}\right], 203$ (34) $\left[\mathrm{C}_{16} \mathrm{H}_{11}^{+}\right], 191$ (26) $\left[\mathrm{C}_{15} \mathrm{H}_{11}^{+}\right], 178(37)\left[\mathrm{C}_{14} \mathrm{H}_{10}^{+*}\right]$.

$$
\mathrm{C}_{34} \mathrm{H}_{36} \mathrm{O} \text { Ber. } 460.2766 \text { Gef. } 460.2760 \text { (MS) }
$$

2) Analog 2) bei der Darstellung von 7, jedoch 20stdg. Erhitzen auf $60^{\circ} \mathrm{C}$ nach Zugabe von Triphenylphosphan und Ausfällen mit Diethylether, aus $0.24 \mathrm{~g}(0.52 \mathrm{mmol})$ 1-[8-(9-Anthryl)-5-methyl1,3,5,7-octatetraen-1-yl]-4-[2-(hydroxymethyl)ethen-1-yl]bicyclo-

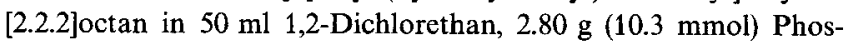
phortribromid in $10 \mathrm{ml}$ 1,2-Dichlorethan, $1.00 \mathrm{~g}$ (3.81 mmol) Triphenylphosphan in $100 \mathrm{ml}$ Toluol; Ausb. $0.20 \mathrm{~g} \mathrm{(49 \% )} 16$ als $(E) /$ (Z)-Gemisch (70:30), Schmp. $>98^{\circ} \mathrm{C}$ (Zers.). - (E)-Isomere: ${ }^{1} \mathrm{H}$ NMR $\left(\mathrm{CDCl}_{3}\right): \delta=8.36\left(\mathrm{~s}, 1 \mathrm{H}, 10^{\prime}-\mathrm{H}\right), 8.33-8.28\left(\mathrm{~m}, 2 \mathrm{H}, 1^{\prime}-, 8^{\prime}-\right.$ H), $8.01-7.95\left(\mathrm{~m}, 2 \mathrm{H}, 4^{\prime}-, 5^{\prime}-\mathrm{H}\right), 7.91-7.60\left(\mathrm{~m}, 15 \mathrm{H}, \mathrm{H}_{\text {Phenyl }}\right)$, $7.47-7.44\left(\mathrm{~m}, 4 \mathrm{H}, 2^{\prime}-, 3^{\prime}-, 6^{\prime}-, 7^{\prime}-\mathrm{H}\right), 7.38$ (d, $\left.1 \mathrm{H}, 1-\mathrm{H}\right), 7.0$ (dd, $\left.J_{2,1}=15.54 \mathrm{~Hz}, J_{2,3}=11.34 \mathrm{~Hz}, 1 \mathrm{H}, 2-\mathrm{H}\right), 6.53(\mathrm{~d}, 1 \mathrm{H}, 3-\mathrm{H})$, $6.34-6.30(\mathrm{~m}, 2 \mathrm{H}, 5-, 6-\mathrm{H}), 6.0\left(\mathrm{dd}, J_{7,8}=15.69 \mathrm{~Hz}, J_{7,6}=9.22 \mathrm{~Hz}\right.$, $1 \mathrm{H}, 7-\mathrm{H}), 5.71(\mathrm{~d}, 1 \mathrm{H}, 8-\mathrm{H}), 5.65\left(\mathrm{~d}, J_{2^{\prime \prime} 1^{\prime \prime}}=15.38 \mathrm{~Hz}, 1 \mathrm{H}, 2^{\prime \prime}-\mathrm{H}\right)$, $5.15\left(\mathrm{dt}, J_{1^{\prime \prime}, \mathrm{CH}_{2}}=7.39 \mathrm{~Hz}, 1 \mathrm{H}, 1^{\prime \prime}-\mathrm{H}\right), 4.78\left(\mathrm{dd}, J_{\mathrm{CH}_{2}, \mathrm{P}}=15.20 \mathrm{~Hz}\right.$, $\left.2 \mathrm{H}, \mathrm{CH}_{2} \mathrm{P}\right), 1.92\left(\mathrm{~s}, 3 \mathrm{H}, \mathrm{CH}_{3}\right), 1.61-1.46\left(\mathrm{~m}, 12 \mathrm{H}, \mathrm{CH}_{2}\right)$. $-\mathrm{MS}$ (EI, $70 \mathrm{eV}): m / z$ (\%) (785.8): $446(1)\left[\mathrm{M}^{+\cdot}-\mathrm{Ph}_{3} \mathrm{P}^{+\cdot} / \mathrm{Br}^{-\cdot}\right], 281$ (1) $\left[\mathrm{C}_{22} \mathrm{H}_{17}^{+\bullet}\right], 262(100)\left[\mathrm{Ph}_{3} \mathrm{P}^{+\bullet}\right], 108(39)\left[\mathrm{C}_{8} \mathrm{H}_{12}^{+\bullet}\right]$.

(Z)-Isomer: ${ }^{1} \mathrm{H}-\mathrm{NMR}\left(\mathrm{CDCl}_{3}\right): \delta=8.41\left(\mathrm{~s}, 1 \mathrm{H}, 10^{\prime}-\mathrm{H}\right), 8.13-8.10$ $\left(\mathrm{m}, 2 \mathrm{H}, 1^{\prime}-, 8^{\prime}-\mathrm{H}\right), 2.03,1.97\left(2 \mathrm{~s}, 3 \mathrm{H}, \mathrm{CH}_{3}\right)$.

Kopplung des TPP-Polyenals 8 mit 16 zu 1-[8-(9-Anthryl)-5-methyl-1,3,5,7-octatetraen-1-yl]-4-\{5-methyl-8-[2-(5,10,15,20-tetraphenyl-21H,23H-porphyrinyl)]-1,3,5,7-octatetraen-1-yl bicyclo[2.2.2]octan (17): Wie bei 10 und 11 beschrieben, jedoch Chromatographie mit Dichlormethan, aus $0.12 \mathrm{~g}(0.15 \mathrm{mmol}) 16$ in $20 \mathrm{ml}$ Diethylether, $0.09 \mathrm{~g}(0.13 \mathrm{mmol}) 8$ in $50 \mathrm{ml}$ 1,2-Dichlorethan und $1.50 \mathrm{ml}(1.50 \mathrm{mmol}) 1 \mathrm{M} \mathrm{BuLi}$ in $10 \mathrm{ml}$ Diethylether; Ausb. $50 \mathrm{mg}$ 
(35\%). Die $(Z)$-Isomeren werden mittels MPLC an Kieselgel mit Hexan/Ethylacetat (94:6) und einem Fluß von $50 \mathrm{ml} / \mathrm{min}$ (Detektion bei $\lambda=257$ bzw. $425 \mathrm{~nm}$ ) abgetrennt. Das $(E)$-Isomer wird mehrmals chromatographiert und aus Dichlormethan/Hexan umkristallisiert; Ausb. $15 \mathrm{mg}(10 \%)$, Schmp. $>270^{\circ} \mathrm{C}$. $-{ }^{1} \mathrm{H}-\mathrm{NMR}$ $\left(\mathrm{CDCl}_{3}\right): \delta=8.90\left(\mathrm{~s}, 1 \mathrm{H}, 3^{\prime \prime \prime}-\mathrm{H}\right), 8.89-8.70\left(\mathrm{~m}, 6 \mathrm{H}, 7^{\prime \prime \prime}-, 8^{\prime \prime \prime}-, 12^{\prime \prime \prime}-\right.$, $\left.13^{\prime \prime \prime}-, 17^{\prime \prime \prime}-, 18^{\prime \prime \prime}-\mathrm{H}\right), 8.36\left(\mathrm{~s}, 1 \mathrm{H}, 10^{\prime}-\mathrm{H}\right), 8.34-8.30\left(\mathrm{~m}, 2 \mathrm{H}, 1^{\prime}-, 8^{\prime}-\right.$ H), $8.24-8.08\left(\mathrm{~m}, 8 \mathrm{H}, o-\mathrm{H}_{\text {Phenyl }}\right), 8.03-7.95\left(\mathrm{~m}, 2 \mathrm{H}, 4^{\prime}-, 5^{\prime}-\mathrm{H}\right)$, $7.88-7.71\left(\mathrm{~m}, 12 \mathrm{H}, m-, p-\mathrm{H}_{\text {Phenyi }}\right), 7.51-7.40\left(\mathrm{~m}, 4 \mathrm{H}, 2^{\prime}-, 3^{\prime}-, 6^{\prime}\right.$, $\left.7^{\prime}-\mathrm{H}\right), 7.35\left(\mathrm{~d}, J_{1,2}=15.70 \mathrm{~Hz}, 1 \mathrm{H}, 1-\mathrm{H}\right), 7.33\left(\mathrm{~d}, J_{1^{n}, 2^{*}}=15.80 \mathrm{~Hz}\right.$, $\left.1 \mathrm{H}, 1^{\prime \prime}-\mathrm{H}\right), 7.33\left(\mathrm{dd}, J_{2^{\prime \prime}, 3^{-}}=10.90 \mathrm{~Hz}, 1 \mathrm{H}, 2^{\prime \prime}-\mathrm{H}\right), 7.02\left(\mathrm{dd}, J_{2,3}=\right.$ $11.39 \mathrm{~Hz}, 1 \mathrm{H}, 2-\mathrm{H}), 6.55(\mathrm{~d}, 1 \mathrm{H}, 3-\mathrm{H}), 6.36\left(\mathrm{~d}, J_{5^{\prime \prime} 6^{\prime \prime}}=16.04 \mathrm{~Hz}\right.$, $\left.1 \mathrm{H}, 5^{\prime \prime}-\mathrm{H}\right), 6.33\left(\mathrm{~d}, J_{5,6}=15.56 \mathrm{~Hz}, 1 \mathrm{H}, 5-\mathrm{H}\right), 6.28\left(\mathrm{~d}, 1 \mathrm{H}, 3^{\prime \prime}-\mathrm{H}\right)$, $6.13\left(\mathrm{dd}, J_{7^{\prime \prime}, 8^{\prime \prime}}=15.43 \mathrm{~Hz}, J_{7^{\prime \prime}, 6^{\prime \prime}}=10.76 \mathrm{~Hz}, 1 \mathrm{H}, 7^{\prime \prime}-\mathrm{H}\right), 6.09(\mathrm{dd}$, $\left.J_{6,6^{\prime \prime}, 55^{\prime \prime}}=16.54 \mathrm{~Hz}, J_{6,6^{\prime \prime}, 7,7^{\prime \prime}}=10.75 \mathrm{~Hz}, 2 \mathrm{H}, 6-, 6^{\prime \prime}-\mathrm{H}\right), 5.83(\mathrm{dd}$, $\left.J_{7,8}=16.48 \mathrm{~Hz}, J_{7,6}=11.84 \mathrm{~Hz}, 1 \mathrm{H}, 7-\mathrm{H}\right), 5.74\left(\mathrm{~d}, 1 \mathrm{H}, 8^{\prime \prime}-\mathrm{H}\right), 5.73$ (d, $1 \mathrm{H}, 8-\mathrm{H}), 2.02\left(\mathrm{~s}, 3 \mathrm{H}, 4^{\prime \prime}-\mathrm{CH}_{3}\right), 1.95\left(\mathrm{~s}, 3 \mathrm{H}, 4-\mathrm{CH}_{3}\right), 1.6-1.31$ (m, $\left.12 \mathrm{H}, \mathrm{CH}_{2}\right),-2.57$ (s, 2H, NH).

$$
\mathrm{C}_{84} \mathrm{H}_{70} \mathrm{~N}_{4} \text { (1135.5) Ber. C 88.85 H } 6.21 \text { N } 4.93
$$

Gef. C 86.30 H 6.27 N 4.62

1,4-Bis [2-(5,5-dimethyl-1,3-dioxan-2-yl)ethen-1-yl]bicyclo[2.2.2]octan (18): In einen Zweihalskolben mit Rückflußkühler gibt man zu $4.65 \mathrm{~g}$ (21.3 mmol) 3, $11.8 \mathrm{~g}$ (113 mmol) 2,2-Dimethyl-1,3propandiol und $20.0 \mathrm{~g}$ Orthoameisensäure-trimethylester in $100 \mathrm{ml}$ Hexan unter Rühren $70.0 \mathrm{mg}(0.40 \mathrm{mmol}) p$-Toluolsulfonsäure. Nach 30 min setzt man festes Natriumcarbonat zu und rührt noch $2 \mathrm{~h}$. Der Feststoff wird über eine Glasfritte abgesaugt und mit Hexan gewaschen. Das Filtrat wird eingeengt, und den Rückstand läßt man in der Kälte auskristallisieren; Ausb. $6.65 \mathrm{~g} \mathrm{(80 \% ),} \mathrm{Schmp.}$ 197-199 ${ }^{\circ} \mathrm{C} .-(E)$-Isomere: ${ }^{1} \mathrm{H}-\mathrm{NMR}\left(\mathrm{CDCl}_{3}\right): \delta=5.79(\mathrm{dd}$, $\left.J_{2,1}=15.92 \mathrm{~Hz}, J_{2,2^{\prime}}=0.56 \mathrm{~Hz}, 2 \mathrm{H}, 2-\mathrm{H}\right), 5.39\left(\mathrm{dd}, J_{1,2^{\prime}}=5.60 \mathrm{~Hz}\right.$, $2 \mathrm{H}, 1-\mathrm{H}), 4.78\left(\mathrm{~d}, 2 \mathrm{H}, 2^{\prime}-\mathrm{H}\right), 3.65-3.46\left(\mathrm{~m}, 8 \mathrm{H}, 4^{\prime}-, 6^{\prime}-\mathrm{H}, \mathrm{AB}-\right.$ System), 1.51 (s, $\left.12 \mathrm{H}, \mathrm{CH}_{2}\right), 1.20,0.72\left(2 \mathrm{~s}, 12 \mathrm{H}, 5^{\prime}-\mathrm{CH}_{3}\right)$.

\section{$\mathrm{C}_{24} \mathrm{H}_{38} \mathrm{O}_{4}$ (390.6) Ber. C 73.81 H 9.81 Gef. C 73.83 H 9.78}

1-[2-(Formyl) ethen-1-yl]-4-[2-(5,5-dimethyl-1,3-dioxan-2-yl)ethen-1-yl Jbicyclo[2.2.2 Joctan (19): In einen Zweihalskolben mit Rückflußkühler gibt man bei Raumtemp. zu $6.65 \mathrm{~g}$ (17.0 mmol) 18 in $100 \mathrm{ml}$ Methanol $/ 3.5 \mathrm{ml}$ Wasser unter Rühren $10 \mathrm{ml}$ Trifluoressigsäure. Nach 30 min werden $10.0 \mathrm{~g}$ Natriumcarbonat zugesetzt, und es wird noch $1 \mathrm{~h}$ gerührt. Der Feststoff wird über eine Glasfritte abgesaugt und mit Diethylether gewaschen. Das Filtrat wird eingeengt und der Rückstand an Kieselgel mit Dichlormethan/Diethyl-

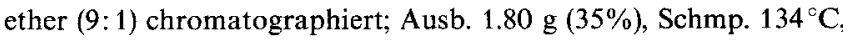
daneben $0.58 \mathrm{~g}(9.0 \%)$ 18. $-(E)$-Isomere: ${ }^{1} \mathrm{H}-\mathrm{NMR}\left(\mathrm{CDCl}_{3}\right): \delta=$ $9.47\left(\mathrm{~d}, J_{\mathrm{CHO}, 1}=7.77 \mathrm{~Hz}, 1 \mathrm{H}, \mathrm{CHO}\right), 6.69\left(\mathrm{~d}, J_{2,1}=15.89 \mathrm{~Hz}, 1 \mathrm{H}\right.$, $2-\mathrm{H}), 5.97(\mathrm{dd}, 1 \mathrm{H}, 1-\mathrm{H}), 5.81\left(\mathrm{dd}, J_{2^{*}, 1^{\prime \prime}}=16.10 \mathrm{~Hz}, J_{2^{*}, 2^{\prime}}=0.61 \mathrm{~Hz}\right.$, $\left.1 \mathrm{H}, 2^{\prime \prime}-\mathrm{H}\right), 5.42\left(\mathrm{dd}, J_{1^{\prime \prime}, 2^{\prime}}=5.50 \mathrm{~Hz}, 1 \mathrm{H}, 1^{\prime \prime}-\mathrm{H}\right), 4.80\left(\mathrm{dd}, 1 \mathrm{H}, 2^{\prime}\right.$ H), 3.66-3.47 (m, 4H, AB-System, 4'-, 6'-H), $1.71-1.51(\mathrm{~m}, 12 \mathrm{H}$, $\left.\mathrm{CH}_{2}\right), 1.21,0.73\left(2 \mathrm{~s}, 6 \mathrm{H}, 5^{\prime}-\mathrm{CH}_{3}\right)$.

$$
\mathrm{C}_{19} \mathrm{H}_{28} \mathrm{O}_{3} \text { (304.4) Ber. C } 74.96 \text { H } 9.27 \text { Gef. C } 75.08 \text { H } 9.40
$$

\section{Umsetzung von 19 zu 5 bzw. 15}

1) a) 1-[4-(9-Anthryl)-1,3-butadien-1-yl]-4-[2-(5,5-dimethyl-1,3dioxan-2-yl)ethen-1-yl]bicyclo[2.2.2]octan: Wie bei 10 und 11 beschrieben, aus $1.8 \mathrm{~g}(5.9 \mathrm{mmol}) 19 \mathrm{in} 50 \mathrm{ml}$ Diethylether, $5.3 \mathrm{~g} \mathrm{(10}$ mmol) 4 in $50 \mathrm{ml}$ Diethylether, $10 \mathrm{ml}(10 \mathrm{mmol})$ einer $1 \mathrm{M}$ Lösung von BuLi in Hexan und durch 20 stdg. Rühren bei Raumtemp.; Ausb. $1.6 \mathrm{~g}(57 \%)$, Schmp. $215-217^{\circ} \mathrm{C}$ (Hexan/Toluol). - (E)-Isomere: ${ }^{1} \mathrm{H}-\mathrm{NMR}\left(\mathrm{CDCl}_{3}\right): \delta=8.34\left(\mathrm{~s}, 1 \mathrm{H}, 10^{\prime}-\mathrm{H}\right), 8.32-8.26(\mathrm{~m}$, $\left.2 \mathrm{H}, 1^{\prime}-, 2^{\prime}-\mathrm{H}\right), 8.0-7.95\left(\mathrm{~m}, 2 \mathrm{H}, 4^{\prime}-, 5^{\prime}-\mathrm{H}\right), 7.47-7.42\left(\mathrm{~m}, 4 \mathrm{H}, 2^{\prime}-\right.$ $\left.3^{\prime}-, 6^{\prime}-, 7^{\prime}-\mathrm{H}\right), 7.26\left(\mathrm{~d}, J_{1,2}=15.55 \mathrm{~Hz}, 1 \mathrm{H}, 1-\mathrm{H}\right), 6.55\left(\mathrm{dd}, J_{2,3}=\right.$ $10.45 \mathrm{~Hz}, 1 \mathrm{H}, 2-\mathrm{H}), 6.36\left(\mathrm{dd}, J_{3,4}=15.20 \mathrm{~Hz}, 1 \mathrm{H}, 3-\mathrm{H}\right), 5.84(\mathrm{~d}$ $\left.J_{2^{\prime \prime}, 1^{\prime \prime}}=15.47 \mathrm{~Hz}, 1 \mathrm{H}, 2^{\prime \prime}-\mathrm{H}\right), 5.75(\mathrm{dd}, 1 \mathrm{H}, 4-\mathrm{H}), 5.45\left(\mathrm{dd}, J_{1^{\prime \prime}, 2^{\prime \prime \prime}}=\right.$ $\left.5.57 \mathrm{~Hz}, 1 \mathrm{H}, 1^{\prime \prime}-\mathrm{H}\right), 4.81$ (d, $\left.1 \mathrm{H}, 2^{\prime \prime \prime}-\mathrm{H}\right), 3.67-3.48\left(\mathrm{~m}, 4 \mathrm{H}, 4^{\prime \prime \prime}-6^{\prime \prime \prime}-\right.$ $\mathrm{H}, \mathrm{AB}-$ System) $1.62-1.61\left(\mathrm{~m}, 12 \mathrm{H}, \mathrm{CH}_{2}\right), 1.22,0.74\left(2 \mathrm{~s}, 6 \mathrm{H}, 5^{\prime \prime \prime}\right.$. $\mathrm{CH}_{3}$ ).

\section{$\mathrm{C}_{34} \mathrm{H}_{38} \mathrm{O}_{2}$ (478.7) Ber. C $85.31 \mathrm{H} 8.00$ Gef. C $85.50 \mathrm{H} 8.09$}

b) 1-[8-(9-Anthryl)-5-methyl-1,3,5,7-octatetraen-1-yl]-4-[2-(5,5dimethyl-1,3-dioxan-2-yl)ethen-1-yl]bicyclo/2.2.2]octan: Aus $1.5 \mathrm{~g}$ (4.9 mmol) 19 in $20 \mathrm{ml}$ Diethylether, $2.9 \mathrm{~g}(5.0 \mathrm{mmol}) 13$ in $50 \mathrm{ml}$ Diethylether, $5.0 \mathrm{ml}(5.0 \mathrm{mmol})$ einer $1 \mathrm{M}$ Lösung von $\mathrm{BuLi}$ in Hexan und durch Deprotonierung bei $0^{\circ} \mathrm{C}$; Ausb. $1.0 \mathrm{~g}(37 \%)$, Schmp. $172{ }^{\circ} \mathrm{C}$ (Petrolether), $-(E) /(Z)$-Isomere: ${ }^{1} \mathrm{H}-\mathrm{NMR}\left(\mathrm{CDCl}_{3}\right)$ : $\delta=8.35\left(\mathrm{~s}, 1 \mathrm{H}, 10^{\prime}-\mathrm{H}\right), 8.33-8.28\left(\mathrm{~m}, 2 \mathrm{H}, 1^{\prime}-, 8^{\prime}-\mathrm{H}\right), 8.0-7.95(\mathrm{~m}$, $\left.2 \mathrm{H}, 4^{\prime}-, 5^{\prime}-\mathrm{H}\right), 7.49-7.43\left(\mathrm{~m}, 4 \mathrm{H}, 2^{\prime}-, 3^{\prime}-, 6^{\prime}-, 7^{\prime}-\mathrm{H}\right), 7.41$ (d, $1 \mathrm{H}, 1-$ H), 7.05 bis $6.92(\mathrm{~m}, 1 \mathrm{H}, 2-\mathrm{H}), 6.65-6.50(\mathrm{~m}, 1 \mathrm{H}, 3-\mathrm{H}), 6.44-6.26$ $(\mathrm{m}, 2 \mathrm{H}, 5-, 6-\mathrm{H}), 6.12-5.65\left(\mathrm{~m}, 3 \mathrm{H}, 2^{\prime \prime}-, 7-, 8-\mathrm{H}\right), 5.47-5.37(\mathrm{~m}$, $\left.1 \mathrm{H}, 1^{\prime \prime}-\mathrm{H}\right), 4.77-4.73$ (m, $\left.1 \mathrm{H}, 2^{\prime \prime \prime}-\mathrm{H}\right), 3.69-3.39$ (m, 4H, 4"'-, $6^{\prime \prime \prime}$ $\mathrm{H}, \mathrm{AB}-\mathrm{System}), 2.09,1.93\left(2 \mathrm{~s}, 3 \mathrm{H}, 4-\mathrm{CH}_{3}\right), 1.58\left(\mathrm{~s}, 12 \mathrm{H}, \mathrm{CH}_{2}\right), 1.23$, $0.73\left(2 \mathrm{~s}, 6 \mathrm{H}, 5^{\prime \prime \prime}-\mathrm{H}\right)$.

\section{$\mathrm{C}_{39} \mathrm{H}_{44} \mathrm{O}_{2}$ (544.8) Ber. C 85.98 H 8.14 Gef. C 85.86 H 7.97}

2) Acetalspaltung: a) Wie bei 19 beschrieben, aus $0.50 \mathrm{~g}(1.04$ mmol) 1-[4-(9-Anthryl)-1,3-butadien-1-yl]-4-[2-(5,5-dimethyl-1,3dioxan-2-yl)ethen-1-yl]bicyclo[2.2.2] octan in $50 \mathrm{ml}$ Dichlormethan $/ 50 \mathrm{ml}$ absol. Ethanol, $10 \mathrm{ml}$ Wasser, $1 \mathrm{ml}$ Trifluoressigsäure und durch Chromatographie an Kieselgel mit Dichlormethan; Ausb. $0.38 \mathrm{~g}(93 \%) 5$.

b) Aus $1.00 \mathrm{~g}$ (1.83 mmol) 1-[8-(9-Anthryl)-5-methyl-1,3,5,7-octatetraen-1-yl]-4-[2-(5,5-dimethyl-1,3-dioxan-2-yl)ethen-1-yl]bicyclo[2.2.2] octan in $50 \mathrm{ml}$ Dichlormethan $/ 50 \mathrm{ml}$ Ethanol, $1 \mathrm{ml}$ Wasser, $20 \mathrm{ml}$ Trifluoressigsäure, bei einer Reaktionszeit von $30 \mathrm{~min}$ und durch Chromatographie an Kieselgel mit Dichlormethan; Ausb. $0.75 \mathrm{~g}(89 \%) 15$.

${ }^{[1]}$ T. Kesmarszky, Dissertation, Univ. Stuttgart, 1990.

${ }^{12]}{ }^{22]}$ H. Kuhn in Biophysik (Hrsg.: W. Hoppe, W. Lohmann, H. Markl, H. Ziegler), Springer, Berlin, 1982, S. 289. - ${ }^{[2 b]}$ G. L. Closs, J. R. Miller, Science 1988, 240, 440-447. - ${ }^{i 2 c 1}$ H. Heitele, P. Finckh, S. Weeren, F. Pöllinger, M. E. Michel-Beyerle, $J$. Phys. Chem. 1989, 93, 5173-5179. - ${ }^{[2 d]}$ J. A. Schmidt, A. R. McIntosh, C. A. Weedon, J. R. Bolton, S. J. Connolly, J. K. Hurley, M. R. Wasielewski, J. Am. Chem. Soc. 1988, 110, 1733-1740. - ${ }^{[2 e]}$ F. C. De Schryver, P. Collart, J. Vandendriessche, R. Goedeweeck, A. Swinnen, M. Van der Auweraer, Acc. Chem. Res. 1987, 20,159-166. - ${ }^{[2]}$ R. W. Munn, Chem. Br. 1984, 20, 518-524.

${ }_{\text {[3] [3a] }}$ M. Calvin, Acc. Chem. Res. 1978, 11, 369-374. - ${ }^{[3 b]} \mathrm{H}$ Kuhn, Phys. Rev. A, 1986, 34, 3409-3425. - ${ }^{33 \mathrm{cl}}$ A. D. Joran, B. A. Leland, M. P. Felker, A. H. Zewail, J. J. Hopfield, P. B. Dervan, Nature (London) 1987, 327, 508-511. - [3d! G. McLendon, Acc. Chem. Res. 1988, 21, 160-167. - ${ }^{[3 \mathrm{e}]} \mathrm{V}$. V. Borovkov, R. P. Evstigneeva, L. N. Strekova, E. I. Filippovich, R. F. Khairutdinov, Usp. Khim. 1989, 58, 1032-1063.

[4] [4a] F. L. Carter, J. Vac. Sci. Technol., B 1983, 1, 959-968. [4b] M. R. Bryce, Annu. Rep. Prog. Chem., Sect. B 1986, 82, 377-394. $-{ }^{[4 \mathrm{c}]}$ D. Haarer, Angew. Chem. Adv. Mater. 1989, 101, 1576-1579. - ${ }^{14 \mathrm{~d}]}$ J. J. Hopfield, J. N. Onuchic, D. N. Beratan, Science 1988, 241, 817-820. - ${ }^{[4 \mathrm{e}]} \mathrm{J}$. M. Lehn, Angew. Chem. 1988, 100, 91-116; Angew. Chem. Int. Ed. Engl. 1988, $27,89-115$.

[5] [5a] F. Effenberger, H. Schlosser, P. Bäuerle, S. Maier, H. Port, H. C. Wolf, Angew. Chem. 1988, 100, 274-277; Angew. Chem. Int. Ed. Engl. 1988, 27, 281-284. - ${ }^{[5 b]}$ S. Maier, H. Port, H. C. Wolf, F. Effenberger, H. Schlosser, Synth. Met. 1989, 29 , E517-E524. - ${ }^{[5 c]}$ B. Heine, E. Sigmund, S. Maier, H. Port, H. C. Wolf, F. Effenberger, H. Schlosser, J. Mol. Electronics 1990, $6,51-60$.

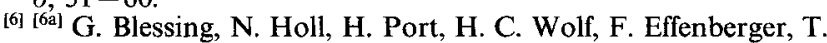
Kesmarszky, H. Schlosser, Mol. Cryst. Liq. Cryst. 1990, 183, $21-30 .-{ }^{[6 b]}$ F. Effenberger, H. C. Wolf, New J. Chem. 1991, $15,117-123$. 
${ }^{[7]}$ F. Effenberger, H. Schlosser, Synthesis 1990, 1085-1094.

${ }^{[8]}{ }^{88 a}$ K. Kumar, S. S. Wang, C. N. Sukenik, J. Org. Chem. 1984, $49,665-670 .-{ }^{[86]}$ E. J. Corey, J. W. Suggs, Tetrahedron Lett. $1975,2647-2650$.

[9] [9a] L. Horner, H. Hoffmann, W. Klink, H. Ertel, V. G. Toscano, Chem. Ber. 1962, 95, 581-601. - ${ }^{[96]}$ W. S. Wadsworth, W. D. Emmons, J. Am. Chem. Soc. 1961, 83, 1733-1738.

${ }^{[10]}$ J. Wolinsky, K. L. Erickson, J. Org. Chem. 1965, 30, 2208 - 2211.

${ }^{[11]}$ G. Benz, Methoden Org. Chem. (Houben-Weyl), 4. Aufl., 1983, Bd. E3, S. $437 \mathrm{ff}$.

[12] [12a] I. M. Goldman, J. Org. Chem. 1969, 34, 1979-1981. - [12b] E. F. Pratt, J. F. Van de Castle, J. Org. Chem. 1961, 26, $2973-2975$.

[13] J. Attenburrow, A. F. B. Cameron, J. H. Chapman, R. M. Evans, B. A. Hems, A. B. A. Jansen, T. Walker, J. Chem. Soc. 1952 , $1094-1111$

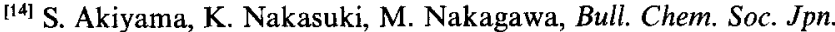
$1971,44,2231-2236$.

${ }^{[15]}$ Y. Takeuchi, S. Akiyama, M. Nakagawa, Bull. Chem. Soc. Jpn. 1972, 45, 3183-3187.

[16] [16a] G. V. Ponomarev, G. B. Maravin, Chem. Heterocycl. Compd. 1982, 18,50-55. - ${ }^{[16 \mathrm{~b}]}$ M. J. Crossley, M. M. Harding, S Sternhell, J. Am. Chem. Soc. 1986, 108, 3608-3613.

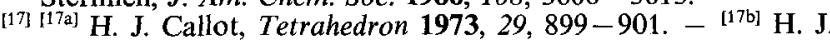
Callot, B. Castro, C. Selve, Tetrahedron Lett. 1978, 2877 - 2880

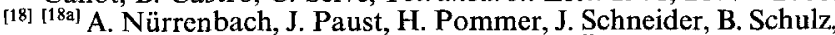
Liebigs Ann. Chem. 1977, 1146-1159. - ${ }^{118 b]}$ H. Pommer, P. C. Thieme, Top. Curr. Chem. 1983, 109, 166-188.

${ }^{[19]}$ C.-P. Niesert, Diplomarbeit, Univ. Stuttgart, 1988.
${ }^{[20]}$ K. Bernhard, G. Englert, H. Mayer, R. K. Müller, A. Rüttimann, M. Vecchi, E. Widmer, R. Zell, Helv. Chim. Acta 1981, 64, $2469-2484$

\section{CAS-Registry-Nummern}

1: 84774-84-5 / 2: 867-13-0 / 3: 140361-36-0 / 4: 33895-18-0 / 5: 129683-19-8 / 6: 140361-37-1 / 7: 140361-38-2 / 8: 140361-39-3/9: $112549-20-9 / 10: 140388-81-4 / 11 \cdot 129713-95-7 / 12: 140361-40-6$ / 13 (Isomer 1): 140361-41-7 / 13 (Isomer 2): 140361-51-9 / 14: 140361-42-8 / 15 (Isomer 1): 140361-43-9 / 15 (Isomer 2): 14046012-4 / 15 (Isomer 3): 140460-13-5 / 16 (Isomer 1): 140361-44-0 / 16 (Isomer 2): 140460-14-6 / 17: 140388-82-5 / 18: 140361-45-1/19: 140361-46-2 / 1,4-Bis[2-(ethoxycarbonyl)ethen-1-yl]bicyclo[2.2.2]octan: 140361-47-3 / 1,4-Bis[2-(hydroxymethyl)ethen-1-yl]bicyclo[2.2.2]octan: 140361-48-4/1-[4-(9-Anthryl)-1,3-butadien-1-yl]-4[2-(hydroxymethyl)ethen-1-yl]bicyclo[2.2.2]octan: 140361-49-5 / (4,4-Dimethoxy-3-methyl-2-butenyl)triphenylphosphoniumchlorid: 53393-73-0 / 2-Formyl-5,10,15,20-tetraphenyl-21 H,23H-porphyrin: 71159-98-3 / 9-Formylanthracen: 642-31-9 / 5-(9-Anthryl)-2methyl-2,4-pentadienol: 140361-50-8 / 1-[8-(9-Anthryl)-5-methyl1,3,5,7-octatetraen-1-yl]-4-[2-(hydroxymethyl)ethen-1-yl]bicyclo[2.2.2]octan: 140361-52-0 / 2,2-Dimethyl-1,3-propandiol: 126-30-7 / 1-[4-(9-Anthryl)-1,3-butadien-1-yl]-4-[2-(5,5-dimethyl-1,3-dioxan-2yl)ethen-1-yl]bicyclo[2.2.2] octan: 140361-53-1 / 1-[8-(9-Anthryl)-5methyl-1,3,5,7-octatetraen-1-yl]-4-[2-(5,5-dimethyl-1,3-dioxan-2-yl)ethen-1-yl]bicyclo[2.2.2]octan (Isomer 1): 140361-54-2 / 1-[8-(9-Änthryl)-5-methyl-1,3,5,7-octatetraen-1-yl]-4-[2-(5,5-dimethyl-1,3-dioxan-2-yl)ethen-1-yl]bicyclo[2.2.2]octan (Isomer 2): 140460-15-7 